\title{
De novo transcriptome profiling uncovers a drastic downregulation of photosynthesis upon nitrogen deprivation in the nonmodel green alga Botryosphaerella sudeticus
}

Deying Sun ${ }^{1 \dagger}$, Jiaqi Zhu ${ }^{1 \dagger}$, Lei Fang ${ }^{1}$, Xin Zhang ${ }^{1}$, Yvonne Chow ${ }^{2}$ and Jianhua Liu ${ }^{1 *}$

\begin{abstract}
Background: Neutral lipid storage is enhanced by nitrogen deprivation (ND) in numbers of green microalgal species. However, little is known about the metabolic pathways whose transcription levels are most significantly altered following ND in green microalgae, especially the nonmodel species.

Results: To start gaining knowledge on this, we performed transcriptome profiling of the nonmodel green microalga Botryosphaerella sudeticus cells in response to ND. Transcriptome of B. sudeticus is de novo assembled based on millions of HiSEQ short sequence reads using CLC Genomics Workbench software. The resulting non-redundant ESTs are annotated based on the best hits generated from the BLASTX homology comparison against the "best" proteins in the model microalgae Chlamydomonas reinhardtii and Chlorella variabilis. By using a pathway-based approach according to KEGG databases, we show that ESTs encoding ribosomal proteins and photosynthetic functions are the most abundantly expressed ESTs in the rapidly growing $B$. sudeticus cells. We find that ESTs encoding photosynthetic function but not the ribosomal proteins are most drastically downregulated upon ND. Notably, ESTs encoding lipid metabolic pathways are not significantly upregulated. Further analyses indicate that chlorophyll content is markedly decreased by 3 -fold and total lipid content is only slightly increased by $50 \%$, consistent with the transcriptional profiling. On the other hand, carbon content and photosynthesis efficiency are only marginally decreased by $7 \%$ and $20 \%$, respectively, indicating that photosynthesis is only slightly reduced upon drastic downregulation of photosynthetic ESTs and chlorophyll content upon ND. In addition, TAG content is found to be greatly increased by 50 -fold, though total lipid content is only slightly increased by 1.5 -fold.

Conclusions: Taken together, our results suggest that light-harvesting proteins and chlorophylls are in excess in B. sudeticus. Degradation of excess photosynthesis proteins is most likely a mechanism for recycling of nitrogen-rich molecules to synthesize new proteins for preparation of gametogenesis and zygospore formation in adaptation and survival upon ND. Furthermore, our analyses indicate that TAG accumulation is largely attributed to the modification of other pre-existing lipid molecules, rather than de novo synthesis. We propose that this is likely an evolutionarily conserved mechanism in many green microalgae species.
\end{abstract}

Keywords: Next-generation sequencing, De novo assembly, Transcriptome profiling, Metabolic pathways, B. sudeticus UTEX2629

\footnotetext{
* Correspondence: liujh@gis.a-star.edu.sg

${ }^{\dagger}$ Equal contributors

'Systems Biology, Genome Institute of Singapore, 60 Biopolis Street, \#02-01,

Singapore 138672, Singapore

Full list of author information is available at the end of the article
} 


\section{Background}

Economic growth is often accompanied by the increase in energy consumption. Depletion of fossil fuel reserves and rising crude oil prices prompt renewed interest in algae biofuel research. Green microalgae are unicellular photosynthetic organisms capable of converting photon energy into chemical energy and assimilating carbon dioxide to form glucose. It has been proposed that microalgae farming could avoid competing with food crops for arable land and fresh water and the energy yield from algae per acre per year would be much higher than other crops [1-3]. In addition, it mitigates greenhouse gas emission. However, current microalgal species and cultivation methodologies are thought to hamper production of algae-based energy in an economically viable manner $[1,4]$.

Many studies have focused on the enhancement of lipid contents in microalgae. Nitrogen deprivation (ND) is one of the most widely used methods to increase lipid storage in algae [4-10]. It is known that ND triggers gametogenesis and zygospore formation in the model green microalga C. reinhardtii [11]. Zygospores can survive under harsh conditions without nutrients for long period of time and ready to revive through germination when external nutrients become available. Storage molecules such as starch and fats (e.g., triacylglycerol or TAG) are the major source of energy essential for spore germination before they are capable of assimilating external nutrients. Therefore, enhancement of lipid storage following ND is at the cost of cell growth arrest and differentiation.

Global transcriptional profiling of microalgal cells in response to ND using next-generation sequencing (or NGS) technologies allows identification of gene regulatory networks involved in adaptation and survival $[7,12]$. Global transcriptional profiling indicates that alteration of lipid metabolic pathways is complex: enhancement of TAG accumulation could be a result of recycling of membrane lipids and de novo glycerolipid biosynthesis [7]. Comparative transcriptome analysis with other algal species may allow gaining insight into molecular mechanisms underlying the metabolic pathways involved in growth arrest and biosynthesis of storage molecules.

Currently, a number of microalgal genome such as Chlamydomonas reinhardtii and Chlorella variabilis are fully sequenced and comprehensively annotated $[13,14]$. Additionally, assembly of genomes and transcriptomes from the millions of short sequence reads generated by NGS technologies has been greatly aided by the use of de Bruijn graph-based sequence-alignment algorithms [15-18]. Several green microalgal transcriptomes have been assembled without reference genomes by using these methodologies [19-21]. However, most of these analyses are primarily focused on gene discovery and metabolic pathway reconstitution.
The green microalga Botryosphaerella sudeticus (UTAX2629) has been previously isolated from a culture of the hydrocarbon-rich slow-growing microalga Botryococcus braunii as a contaminant [22]. Phylogenetic analysis based on rDNA sequence indicates that $B$. sudeticus is more related to Characiopodium hindakii rather than B. braunii [23]. Observation of gametes leads to the suggestion that $B$. sudeticus may have sexual life cycle [24]. We showed that the maximal cell density of $B$. sudeticus cultures was 2 -fold of that of $C$. reinhardtii under conditions of phototrophic growth, suggesting a useful candidate for algal biomass production. Gametogenesis and lipid accumulation were induced by ND. To investigate transcriptional alteration of metabolic pathways following ND, we performed global transcriptional profiling of B. sudeticus cells in response to ND based on the transcriptome assembled without reference genome. By using a pathway-based approach, we show that ESTs encoding photosynthetic function and ribosomal proteins are the most abundant ESTs in rapidly growing $B$. sudeticus cells. Upon ND, photosynthetic function-encoding but not ribosomal proteinencoding ESTs are drastically downregulated, suggesting a programmed cellular response for differentiation. This transcriptional response to ND is found to be conserved in C. reinhardtii, possibly in most green microalgae.

\section{Results}

Gametogenesis and lipid accumulation are enhanced by nitrogen deprivation (ND) in $B$. sudeticus

$B$. sudeticus (UTEX2629) was often found to overgrown in cultures of B. braunii (UTEX572) (Sun and Liu, unpublished data). It was partly because of the slow growth rate of $B$. braunii. To investigate how well $B$. sudeticus grew, we tested its phototrophic growth in media of HS [25], $\mathrm{BB}$ [26], and 2x BB in shaking flask with bubbling of $\mathrm{CO}_{2}$ under constant light (i.e., $250 \mu \mathrm{mol}$ photon $\mathrm{m}^{-2} \mathrm{sec}^{-1}$ ). We compared the growth curve of B. sudeticus with that of $C$. reinhardtii, a best-studied green microalga. The growth rate and the maximal cell density of $B$. sudeticus and $C$. reinhardtii were very similar in HS medium (Figure 1A). On the other hand, though the growth rate of $B$. sudeticus remained to be similar as that of $C$. reinhardtii, the maximal cell density of $B$. sudeticus was increased by $\sim 25 \%$ in $1 \mathrm{xBB}$ medium and nearly by $100 \%$ (or 2-fold) in 2x BB medium compared to that of $C$. reinhardtii. This result suggests that $B$. sudeticus is a useful strain for potential microalgal biomass production.

We subsequently investigate if gametogenesis would be induced by ND. We noted that there were $\sim 1 \%$ of gametes in log-phase growth culture (Figure 1B; upper panel). On the other hand, $\sim 10 \%$ of gametes were found three days after ND. Though we did not obtain the cell-cell fusion event in EM analysis (Data not shown), aggregation and precipitation of cells upon ND was apparent (Figure 1B; 

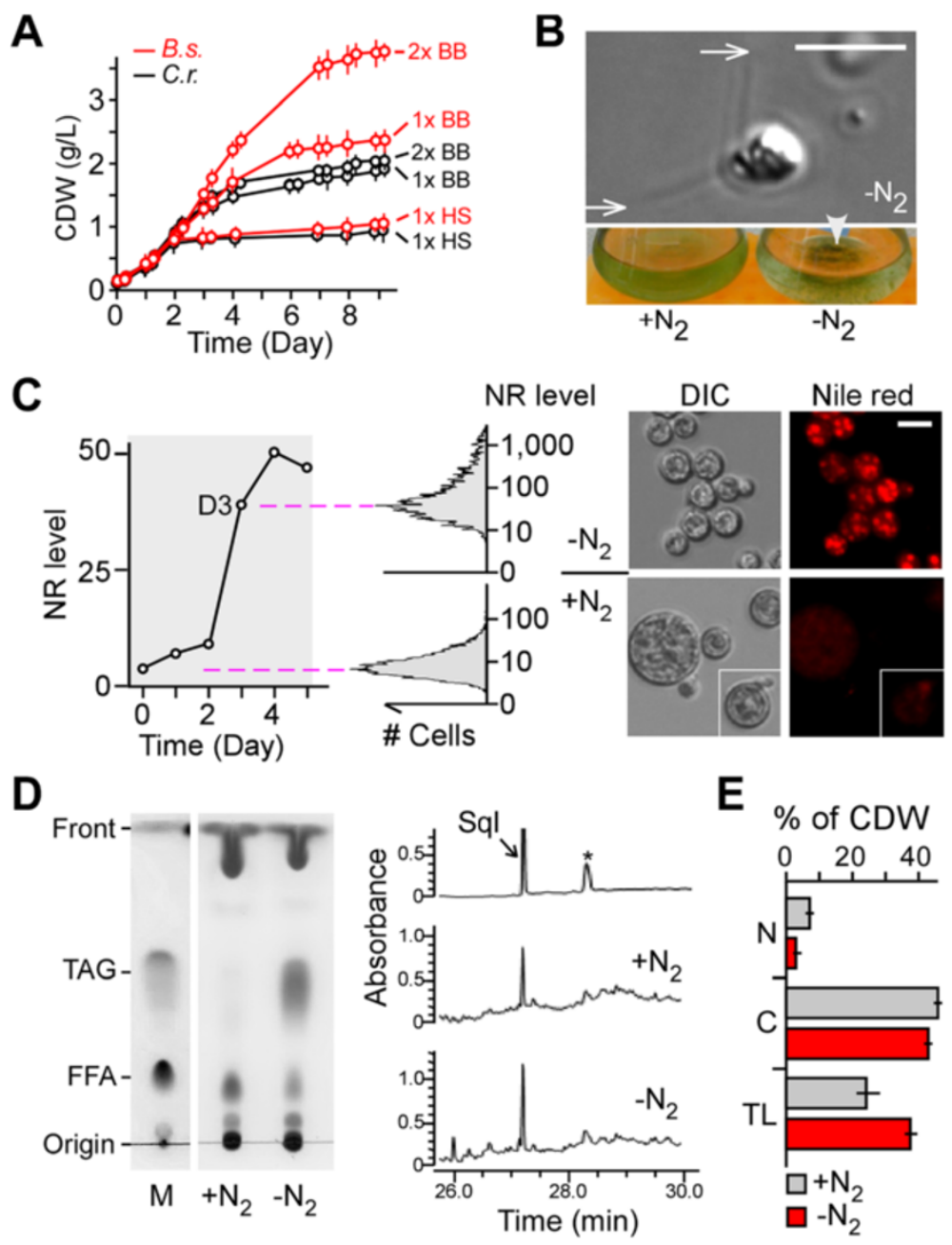

Figure 1 Characterization of growth, gametogenesis, and lipid content in B. sudeticus. (A) B. sudeticus culture reaches relatively high maximal cell density. Growth curves of B. sudeticus (in red) and C. reinhardtii (in black) in various media indicated are shown. X-and Y-axis indicate time (in day) and cell dry weight (CDW; in $\mathrm{g} / \mathrm{L}$ ), respectively. HS and BB stand for High Salt and Bold-modified Bristol media, respectively. Error bars represent the standard deviation of triplicate samples. (B) Gametogenesis is induced by ND in B. sudeticus. Upper panel shows a gamete whose tip of flagella is denoted by an arrow. Bottom panel shows part of conical flasks in which sedimented cell aggregates are denoted by an arrow-head. Medium with nitrogen $\left(+\mathrm{N}_{2}\right)$ or without nitrogen $\left(-\mathrm{N}_{2}\right)$ is indicated. (C) Lipid accumulation is enhanced by ND in B. sudeticus. Left panel shows the increased intensity of Nile red signal in cells after ND. X-and Y-axis indicate the time (in day) and Nile red signal level (in arbitrary units), respectively. Nile red signal level of cell samples are based on the median level derived from FACS analysis as shown in the mid panel. Right panel shows the Nile red stained cells prior to $\left(+\mathrm{N}_{2}\right)$ and after $\left(-\mathrm{N}_{2}\right) \mathrm{ND}$. (D) Level of triacylglycerol (TAG) and squalene is increased in B. sudeticus following ND. Left panel shows the TLC analysis and right panel shows the GC-MS analysis (MS result is not shown) of total lipid in B. sudeticus prior to $\left(+\mathrm{N}_{2}\right)$ and after $\left(-\mathrm{N}_{2}\right)$ ND. (E) Contents of nitrogen $(\mathrm{N})$, carbon $(\mathrm{C})$, and total lipid $(\mathrm{TL})$. Bar-plots show the level of $N$, $C$, and $T L$ against CDW in nitrogen-replete $\left(+\mathrm{N}_{2}\right)$ and nitrogen-starved $\left(-\mathrm{N}_{2}\right)$ cells.

bottom panel). Hence, we concluded that ND induced gametogenesis in B. sudeticus.

To examine whether ND would enhance lipid storage, nitrogen-deprived (ND) cells were stained with a lipophilic dye Nile red and subjected to FACS analysis (Figure 1C). Average level of Nile red signals in cells 3 days after ND was increased by 40 -fold compared to that prior to ND. TLC and GC-MS analyses indicated that TAG and squalene (SQL) contents (i.e., \% of $\mathrm{CDW}$ ) were increased by $\sim 50$-fold and 2.5 -fold compared to that of nitrogen-replete cells (i.e., TAG, $3.74 \pm 0.14 \%$ versus $0.08 \pm 0.01 \%$ of CDW, p-value $=1.5 \mathrm{e}-06$; SQL, $0.15 \pm 0.01$ versus $0.06 \pm 0.01 \%$ of $C D W, \mathrm{p}$-value $=6.4 \mathrm{e}-05)$, respectively (Figure 1D; Additional file 1: Table S8). Notably, total lipid of the early log-phase cells exhibited the relatively low level of TAG content. 
Actively growing $B$. sudeticus cultures were maintained by subculturing or 8-fold dilution in fresh $\mathrm{BB}+\mathrm{N}$ medium prior to exhaustion of nitrate in the medium (i.e., $3.8 \pm 0.3 \mathrm{mg} / \mathrm{L}$ nitrate at day 3 prior to dilution/ subculturing). We noted that the cell mass continued to increase under ND conditions (i.e., CDW from $0.14 \pm 0.03$ $\mathrm{g} / \mathrm{L}$ at day 0 to $0.94 \pm 0.06 \mathrm{~g} / \mathrm{L}$ at day 3 under ND conditions). To examine the alteration of nitrogen $(\mathrm{N})$ and carbon (C) contents in cells after ND, we performed elemental analysis of $\mathrm{N}$ and $\mathrm{C}$ in cells. It was clear that $\mathrm{N}$ contents in the ND cells was greatly dropped by $2.5-$ fold compared to that of nitrogen-replete cells (i.e., $2.8 \pm$ $0.09 \%$ versus $7.14 \pm 0.16 \%$ of CDW, $\mathrm{p}$-value $=2.16 \mathrm{E}-06$ ) (Figure 1E). On the other hand, the $C$ content in the ND cells was only slightly reduced compared to that of nitrogen-replete cells (i.e., $42.7 \pm 0.31 \%$ versus $45.9 \pm 0.46 \%$ of CDW). This result suggests that carbon assimilation is not strongly affected upon ND for 3 days. While TAG content were drastically increased by 50 -fold upon ND stress (i.e., $3.47 \pm 0.14 \%$ versus $0.08 \pm 0.01 \%$ of CDW; p-value $=1.5 \mathrm{e}-06$ ), total lipid (TL) content in ND cells was only slightly increased by 1.54 -fold compared to that of nitrogen-replete cells (i.e., $37.37 \pm 1.42 \%$ versus $24.21 \pm 2.71 \%$ of CDW). This result indicates that majority of the storage TAG molecules in ND cells are accumulated through the modification of pre-existing lipids, rather than de novo synthesis.

\section{De novo assembly and annotation of the $B$. sudeticus transcriptome}

For analysis of transcriptional profiling of B. sudeticus cells in response to ND, we first wanted to assemble the transcriptome without reference genome. For this reason, cDNA libraries were constructed from the ND and nitrogen-replete cells. A total of 52,000,000 paired-reads (90-bp in length) pooled from both growth conditions were subjected to de novo transcriptome assembly using the CLC Genomics Workbench software (see Methods). To this end, 56,000 non-redundant contigs/ scaffolds/ ESTs (ESTs were used hereafter) were generated. Length of ESTs ranged from 200 to 15,487 bps with a median of 369 bps or average of 660 bps (Figure 2A). Approx. 90\% of all reads in either growth condition was mapped to the ESTs. Absolute level of ESTs was normalized to RPKM (Reads Per Kilobase per Million mapped reads) ranged from 0.09 to 17,293 with a median of 2.21 (Figure 2B).

B. sudeticus ESTs were annotated based on the best-hit proteins generated by sequence homology comparison using the Basic Local Alignment Search Tool BLASTX suite (http://blast.ncbi.nlm.nih.gov) against the "best" proteins in the model green microalgae $C$. reinhardtii and $C$. variabilis $[13,14]$ with a cutoff expectation-value of $1 \mathrm{E}-06$. As a result, a total of 7,625 (13.6\%) non-redundant ESTs in B. sudeticus were annotated, of which, 6,559 ESTs had best-hits in $C$. reinhardtii and 1,066 in C. variabilis $[13,14]$ (Additional file 2: Table S1).

Gene Ontology (GO) [27] and KEGG Ortholog (KO) [28] annotations that associated with the best-hit proteins in $C$. reinhardtii and $C$. variabilis were extracted for transcriptome analysis in B. sudeticus. Of a total of 7,625 annotated ESTs, 4,860 were found to be associated with at least one of the 2,122 GO-terms (i.e., 433 terms in Biological Processes, 152 terms in Cellular Components, and 1,537 terms in Molecular Functions). GO terms associated with the highest number of ESTs in three categories were metabolism (322 ESTs; biological process), membrane (361 ESTs; cellular component), and ATP-binding (744 ESTs; molecular function). The top 10 GO-terms in three categories were shown in Figure $2 \mathrm{C}$ (Additional file 3: Table S2).

Out of 7,625 ESTs, 1,129 were found to be associated with at least one of the 114 KEGG metabolic pathways [28]. Top 30 KEGG metabolic pathways were shown in Figure 2D (Additional file 4: Table S3). We took a pathway-based approach to study the coherence of ESTs absolute abundances in the rapidly growing cells and the coherence of ESTs abundance alterations (or ratios) upon ND in B. sudeticus (see below).

\section{Overview of the pathway-based transcriptome analysis}

Based on KEGG databases, we focused on the coherence analysis of the absolute abundance (i.e., steady-state level in nitrogen-replete cells) and relative abundance (i.e., ratio between levels in ND and nitrogen-replete cells) of a subset of the metabolic pathway-associated ESTs. We assumed that co-regulated basal or constitutive transcription of the pathway-associated ESTs would display a high consistency in EST levels in rapidly growing cells. Likewise, co-regulated transcriptional alteration in response to ND would exhibit a high coherence of the pathway-associated EST ratios between levels in ND and nitrogen-replete cells.

We used the occurrence density of the pathwayassociated ESTs in a range that contained the first 50\%, middle $50 \%$, or second $50 \%$ of the ESTs based on ranks to approximate the transcriptional coherence of a given metabolic pathways (see Methods). Coherence of the constitutive transcription and ND-induced transcription of the pathway-associated ESTs was determined based on ranks by the absolute abundance and relative abundance or ratio, respectively. A metabolic pathway was designated as a co-transcriptionally regulated (or coherent) pathway if the occurrence density of the pathway-associated ESTs in one of the three half ranges was 2-fold above background and the p-value was less than 0.05 based on ranks by level (i.e., for basal transcription) or ratio (i.e., for transcriptional response). We also tested the occurrence density in the top $10 \%$ of the most abundant ESTs based on ranks by level or in the top (or bottom) $10 \%$ of the most 


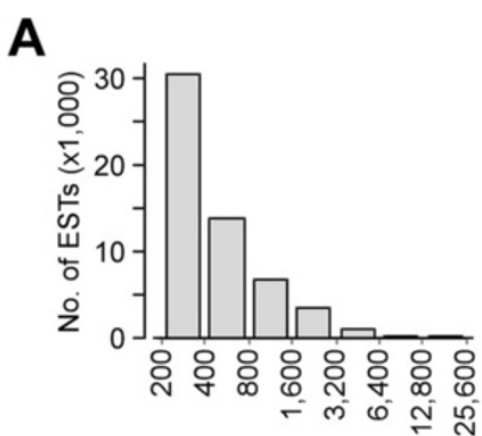

Bin of EST length (bp)
B

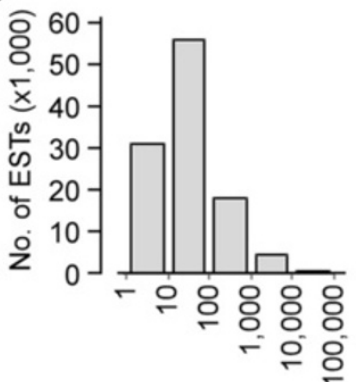

Bin of EST level (RPKM)

\section{C}

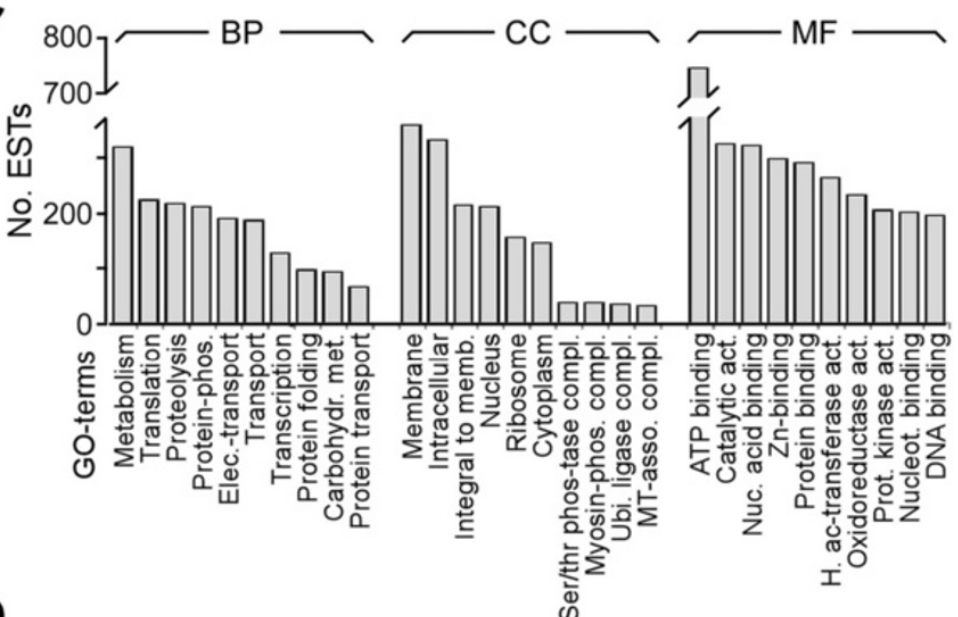

D

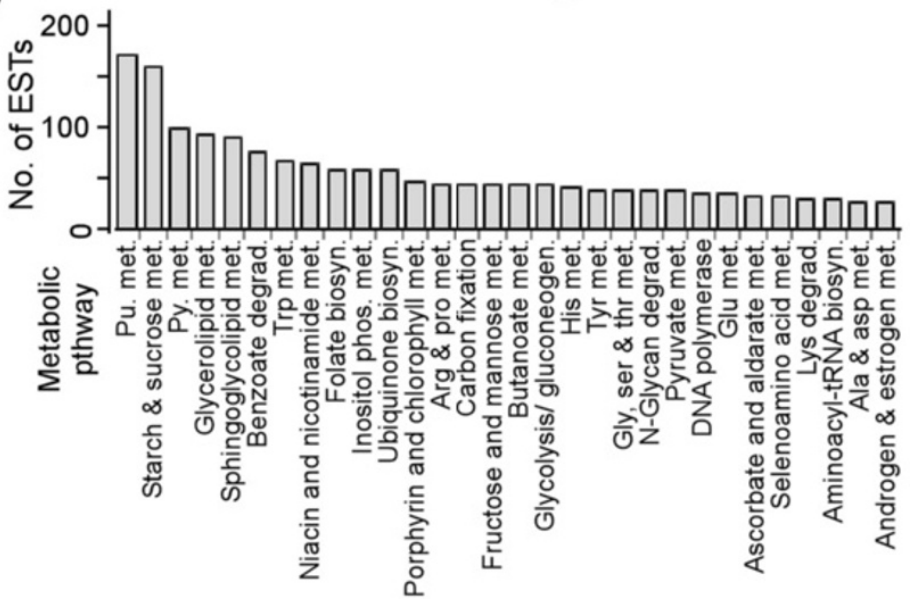

Figure 2 Characteristics of the B. sudeticus transcriptome. A total of 56 thousand ESTs is assembled based on 53 million paired-end reads (90 bp in length), of which, 7,600 ESTs were annotated. (A) Length distribution of all ESTs. X-and Y-axis indicate the binned EST length and the number of ESTs in each bin, respectively. (B) Level distribution of all ESTs. Levels are normalized to RPKM. X-and Y-axis indicate the binned EST level (in RPKM) and the number of ESTs in each bin, respectively. (C) List of GO-terms that are associated with ESTs annotations. Top 10 GO-terms in each category are shown. X-axis indicates the GO-terms and Y-axis indicates the number of ESTs associated with the GO-terms. BP, CC, and MF stand for biological process, cellular component, and molecular function, respectively. (D) List of KEGG metabolic pathways that are associated with ESTs annotations. Top 30 metabolic pathways are listed. The display is identical to (C). 
upregulated (or downregulated) ESTs based on ranks by ratio (e.g., relative occurrence density $>2$-fold above background and $\mathrm{p}$-value $<0.05$ ) for coherently regulated metabolic pathways (see Methods).

We proposed that coherent pathways were potentially co-transcriptionally regulated. Given that the magnitude of alteration in transcription levels following ND was a function of time, magnitude of change in transcription levels would vary in cells sampled at different time point after stress. On the other hand, coherence of transcriptional response for metabolic pathways would be remained throughout the course of response.

\section{Coherent transcription of the ribosomal protein-encoding} ESTs in $B$. sudeticus

Absolute level of the 7,625 annotated ESTs in B. sudeticus was approximated by RPKM (see Methods). We found that 16 out of top 30 most abundant ESTs encoded putative ribosomal proteins, indicating that RP-encoding ESTs are enriched in the topmost expressed ESTs compared to background (i.e., 53.3\% versus $1.29 \%$, p-value $<1.33 \mathrm{E}-07$ ) in actively growing $B$. sudeticus cells (Table 1 ). This result is consistent with a notion that protein synthesis is one of the most active functions in actively growing cells [29].

Cytosolic or eukaryotic ribosomal proteins (i.e., eRPs) are encoded by nuclear genes in green microalgae. On the other hand, chloroplast and mitochondrion or archaea ribosomal proteins (i.e., aRPs) are encoded by both the nuclear and chloroplast or mitochondrion genes, respectively. Transcriptional coordination would be relatively easy to achieve for a group of genes localized in a single genome compared to those in two genomes such as nuclear and chloroplast or mitochondrial genomes. Hence, we hypothesized that the transcription consistency of the eRP-encoding ESTs would be high compared to that of aRP-encoding ESTs. To test this hypothesis, we first determined the relative occurrence density in the 3 half-ranges (i.e., first, middle, and second 50\%) of the eRP- and aRPencoding ESTs (i.e., nuclear encoded aRP genes) based on the rank by abundance (Figures $3 \mathrm{~A}$ and $3 \mathrm{~B}$ ). It was clear that the relative occurrence densities in the first and middle halves of the eRP-encoding ESTs were much higher than background (i.e., 56.8-fold and 36.1-fold above background; p-value $<0.001$ ), indicating that $75 \%$ of the eRP-encoding ESTs displayed a coordinated level of constitutive transcription. On the other hand, the relative occurrence density only in the range of the first $50 \%$ of the aRP-encoding ESTs was higher than background (i.e., 7.2-fold above background; p-value $<0.001$ ).

We noted that the occurrence density of both eRP- and aRP-encoding ESTs was clearly enriched in the top 10\% of the most abundant ESTs in actively growing B. sudeticus cells (i.e., 9.9-fold and 5.7-fold above background; p-value $<0.001)$, indicating that protein synthesis is highly active in both cytoplasm and chloroplast/ mitochondrial to support growth of B. sudeticus cells.

We subsequently examined the relative occurrence density of the eRP- and aRP-encoding ESTs based on ranks by ratio between levels in ND and nitrogen-replete cells. Occurrence density of the eRP-encoding ESTs was found to be enriched in the range containing the middle half and the second half of the eRP-encoding ESTs (i.e., 3.24-fold and 2.29-fold above background; p-value < 0.001 ), suggesting that change in transcription levels of eRP-encoding ESTs is consistent during response to ND (Figure $3 \mathrm{C}$ ). On the other hand, occurrence density in all 3 ranges of aRP-encoding ESTs was not enriched. This result supports our hypothesis that co-transcriptional regulation of genes localized in a single genome is relatively easy to achieve compared to those located in different genomes. We noted that transcription of RP genes was not significantly downregulated in B. sudeticus cells 3 days after ND (Figure 3D).

\section{Energy metabolism-associated ESTs appear to be most abundant and highly coherent in actively growing $B$. sudeticus cells}

We examined the consistency in levels of ESTs associated with various metabolic pathways in actively growing $B$. sudeticus cells. To this end, 66 KEGG metabolic pathways were found to associate with at least 10 ESTs based on annotation of the B. sudeticus transcriptome (Additional file 5: Table S4). The eRP- or aRP-encoding ESTs were included as control. Relative occurrence density of individual metabolic pathways in ranges containing the first, middle, and second halves of the pathway-associated ESTs based on ranks by level was determined (Figure 4A). Fourteen out of the 66 metabolic pathways exhibited high relative occurrence density in one of the 3 ranges of the pathway-associated ESTs based on ranks by level (i.e., 2fold above background; p-value $<0.05$ ), 5 of which were found to be enriched in the top $10 \%$ of the most abundant ESTs (i.e., 2-fold above background; p-value < 0.05).

Coherent transcription was found in 5 carbohydrate, 2 lipid, 2 amino acid, and 5 energy metabolic pathways (Figure 4B). Of the 14 coherently transcribed pathways, 5 were enriched in the top $10 \%$ of the most abundant ESTs: 2 involved in carbohydrate metabolisms (glycolysis/ gluconeogenesis and pentose phosphate pathway) and 3 involved in energy metabolisms (carbon fixation, oxidative phosphorylation, and photosynthesis). Glycolysis is the process of converting glucose into pyruvate and generating small amounts of ATP (energy) and NADH (reducing power) molecules; and the pentose phosphate pathway is a process of glucose turnover that produces NADPH and pentoses (for nucleic acid) [30]. Both were primary metabolism and essential for cell growth. Photosynthesis (i.e., light-dependent photosynthetic reaction) is the process 
Table 1 List of the top 30 most abundant ESTs in B. sudeticus

\begin{tabular}{|c|c|c|c|c|c|c|}
\hline $\mathrm{Rk}^{\mathrm{a}}$ & EID $^{\mathbf{b}}$ & Level & Ratio & Genome & $\mathrm{PID}^{c}$ & Description $^{d}$ \\
\hline 1 & 3556 & 4.03 & -0.50 & Chlre4 & 376403 & \\
\hline 2 & 318 & 3.98 & -0.24 & Chlre4 & 322197 & $\begin{array}{l}\text { RP-S29e, RPS29; small } \\
\text { subunit ribosomal } \\
\text { protein S29e }\end{array}$ \\
\hline 3 & 5 & 3.81 & 0.20 & Chlre4 & 395228 & \\
\hline 4 & 36952 & 3.78 & 0.16 & ChINC64A & 28889 & \\
\hline 5 & 106 & 3.70 & -0.04 & Chlre4 & 155068 & \\
\hline 6 & 57 & 3.69 & 0.18 & Chlre4 & 407233 & $\begin{array}{l}\text { Glucan1,4-alpha- } \\
\text { glucosidase. }\end{array}$ \\
\hline 7 & 8 & 3.67 & -0.21 & Chlre4 & 195592 & $\begin{array}{l}\text { RP-S20e, RPS20; small } \\
\text { subunit ribosomal } \\
\text { protein S20e }\end{array}$ \\
\hline 8 & 520 & 3.65 & -0.03 & Chlre4 & 195131 & $\begin{array}{l}\text { RP-L22e, RPL22; large } \\
\text { subunit ribosomal } \\
\text { protein L22e }\end{array}$ \\
\hline 9 & 127 & 3.62 & 0.05 & Chlre4 & 418234 & \\
\hline 10 & 142 & 3.61 & -0.06 & Chlre4 & 128309 & $\begin{array}{l}\text { RP-L44e, RPL44; large } \\
\text { subunit ribosomal } \\
\text { protein L44e }\end{array}$ \\
\hline 11 & 512 & 3.56 & -0.12 & Chlre4 & 194928 & $\begin{array}{l}\text { RP-L38e, RPL38; large } \\
\text { subunit ribosomal } \\
\text { protein L38e }\end{array}$ \\
\hline 12 & 181 & 3.50 & 0.10 & Chlre4 & 127247 & $\begin{array}{l}\text { RP-L35Ae, RPL35A; } \\
\text { large subunit ribosomal } \\
\text { protein L35Ae }\end{array}$ \\
\hline 13 & 633 & 3.45 & -0.33 & Chlre4 & 206640 & $\begin{array}{l}\text { rbcS; ribulose- } \\
\text { bisphosphate } \\
\text { carboxylase small } \\
\text { chain[EC:4.1.1.39] }\end{array}$ \\
\hline 14 & 316 & 3.41 & 0.00 & Chlre4 & 129742 & $\begin{array}{l}\text { RP-S21e, RPS21; small } \\
\text { subunit ribosomal } \\
\text { protein S21e }\end{array}$ \\
\hline 15 & 31 & 3.38 & 0.05 & Chlre4 & 57302 & $\begin{array}{l}\text { RP-S25e, RPS25; small } \\
\text { subunit ribosomal } \\
\text { protein S25e }\end{array}$ \\
\hline 16 & 9 & 3.30 & -0.26 & Chlre4 & 166012 & $\begin{array}{l}\text { RP-L30e, RPL30; large } \\
\text { subunit ribosomal } \\
\text { protein L30e }\end{array}$ \\
\hline 17 & 311 & 3.26 & 0.09 & Chlre4 & 191758 & $\begin{array}{l}\text { RP-L32e, RPL32; large } \\
\text { subunit ribosomal } \\
\text { protein L32e }\end{array}$ \\
\hline 18 & 1207 & 3.26 & 0.16 & ChINC64A & 144034 & \\
\hline 19 & 114 & 3.23 & -0.33 & Chlre4 & 344365 & \\
\hline 20 & 590 & 3.19 & -0.18 & Chlre4 & 195601 & $\begin{array}{l}\text { RP-S17e, RPS17; small } \\
\text { subunit ribosomal } \\
\text { protein } \mathrm{S17e}\end{array}$ \\
\hline 21 & 1921 & 3.18 & 0.21 & Chlre4 & 195587 & $\begin{array}{l}\text { RP-L11e, RPL11; large } \\
\text { subunit ribosomal } \\
\text { protein L11e }\end{array}$ \\
\hline 22 & 287 & 3.17 & -0.06 & Chlre4 & 292191 & \\
\hline 23 & 282 & 3.16 & -0.41 & Chlre4 & 195585 & $\begin{array}{l}\text { RP-L10Ae, RPL10A; } \\
\text { large subunit ribosomal } \\
\text { protein } L 10 A e\end{array}$ \\
\hline
\end{tabular}

Table 1 List of the top $\mathbf{3 0}$ most abundant ESTs in B. sudeticus (Continued)

\begin{tabular}{|c|c|c|c|c|c|c|}
\hline 24 & 50 & 3.16 & -0.84 & Chlre4 & 156131 & $\begin{array}{l}\text { TC.AMT; ammonium } \\
\text { transporter, Amt family }\end{array}$ \\
\hline 25 & 20 & 3.15 & 0.45 & Chlre4 & 120079 & $\begin{array}{l}\text { SIRT2, SIR2L2; NAD- } \\
\text { dependent deacetylase } \\
\text { sirtuin } 2 \text { [EC:3.5.1.-]; in } \\
\text { linear amides. }\end{array}$ \\
\hline 26 & 1000 & 3.14 & -0.26 & Chlre4 & 126853 & $\begin{array}{l}\text { RP- } L 37 \mathrm{Ae}, \mathrm{RPL} 37 \mathrm{~A} ; \\
\text { large subunit ribosomal } \\
\text { protein } \mathrm{L} 37 \mathrm{Ae}\end{array}$ \\
\hline 27 & 480 & 3.14 & -0.16 & Chlre4 & 78109 & $\begin{array}{l}\text { RP-L31e, RPL31; large } \\
\text { subunit ribosomal } \\
\text { protein L31e }\end{array}$ \\
\hline 28 & 296 & 3.12 & -0.24 & Chlre4 & 36709 & \\
\hline 29 & 278 & 3.12 & 0.01 & Chlre4 & 145271 & $\begin{array}{l}\mathrm{RP}-\mathrm{L} 14 \mathrm{e}, \mathrm{RPL} 14 \text {; large } \\
\text { subunit ribosomal } \\
\text { protein L14e }\end{array}$ \\
\hline 30 & 545 & 3.11 & -0.07 & Chlre4 & 153674 & \\
\hline
\end{tabular}

Note: a, rank; b, EST ID; c, Protein ID in C. reinhardtii or C. variabilis; d, EST function based on its best hit protein.

of harvesting light energy and generating ATP and $\mathrm{NADH}$ which in turn utilized to reduce carbon dioxide to organic molecules in a process known as carbon fixation (or light-independent photosynthetic reaction). Oxidative phosphorylation is the process of transferring electrons to oxygen and generating ATP in mitochondria. Majority ATP in cells were generated through oxidative phosphorylation [30]. Hence, we concluded that photosynthesis and energy metabolisms are the most active functions required for rapidly growth of $B$. sudeticus.

\section{Transcription of photosynthetic ESTs is most drastically downregulated upon ND in B. sudeticus}

To investigate metabolic pathways that exhibited cotranscriptional alteration in response to ND, the relative occurrence density of the 66 metabolic pathways in the 3 ranges (i.e., the first, middle, and second halves) of the pathway-associated ESTs was determined based on ranks by ratio between levels in ND and nitrogen-replete cells (Figure 5A). Five out of the 66 metabolic pathways such as glutathione metabolism, ATP synthesis, oxidative phosphorylation, photosynthesis, and riboflavin metabolism exhibited coherent response to ND (Figure 5B). It was interesting that 4 of the 5 coherently responsive metabolic pathways displayed a consistency in constitutive transcription (see Figure 4B). On the other hand, of the 5 metabolic pathways exhibiting coherent response, only photosynthesis showed to be downregulated by $43.8 \%$ based on the median of photosynthesis-associated ESTs following ND (i.e., p-value < 0.001) (Figure 5C). Median level of the remaining 4 pathways was hardly altered. Photosynthesis was the only pathway whose associated ESTs were enriched in the top $10 \%$ of the most 

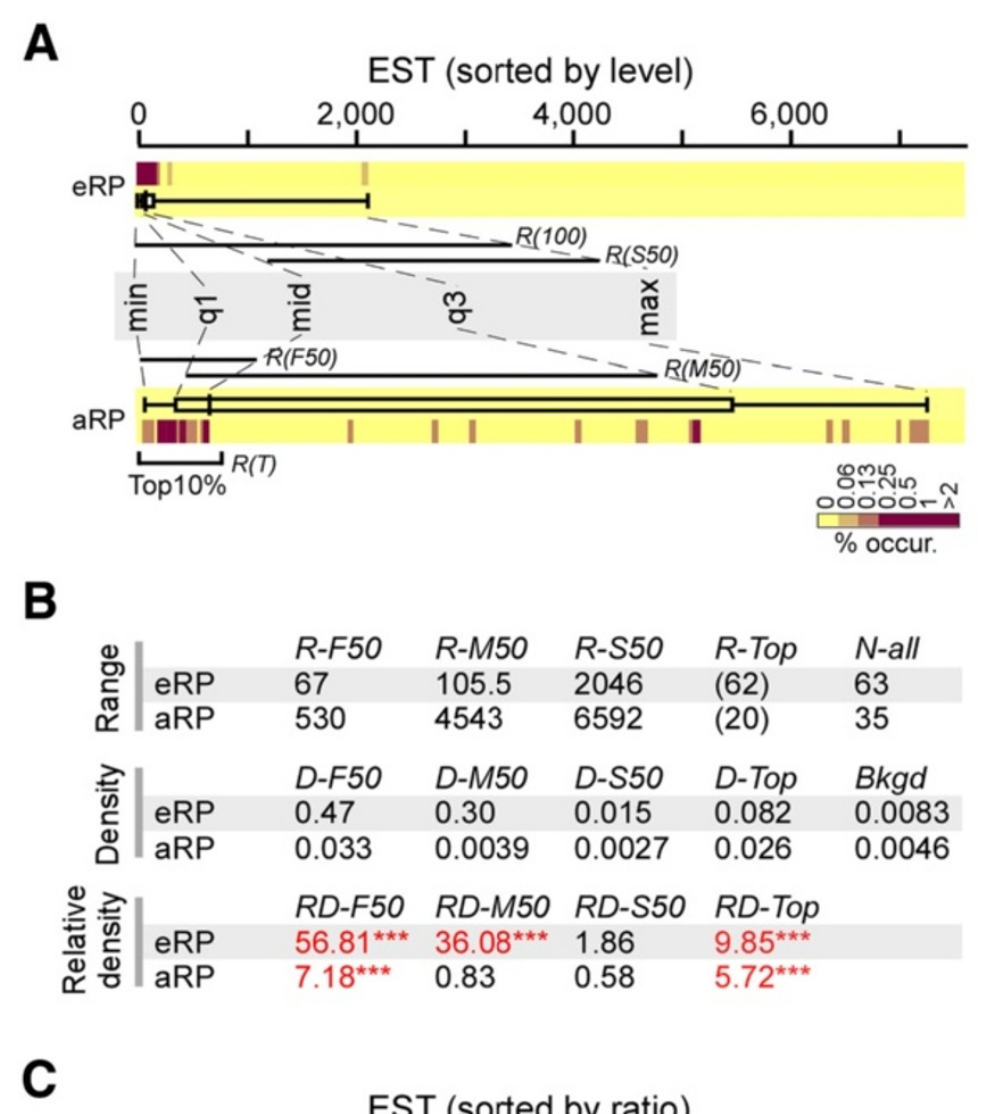

EST (sorted by ratio)
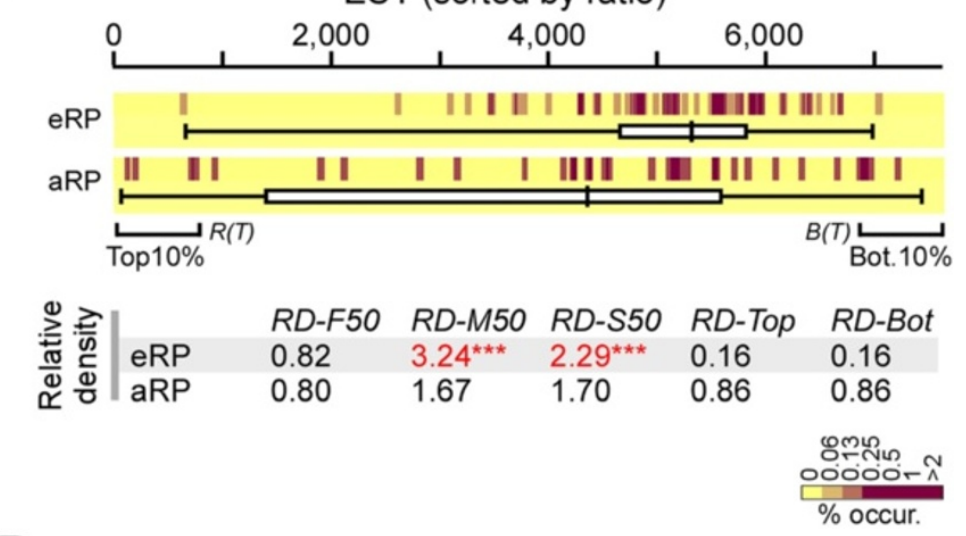

D

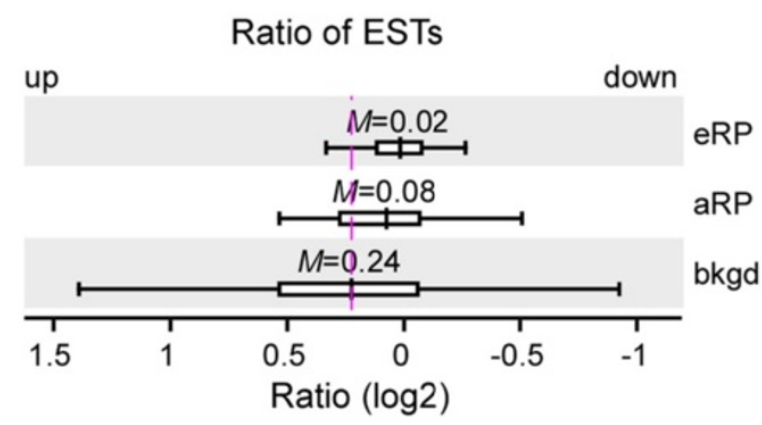




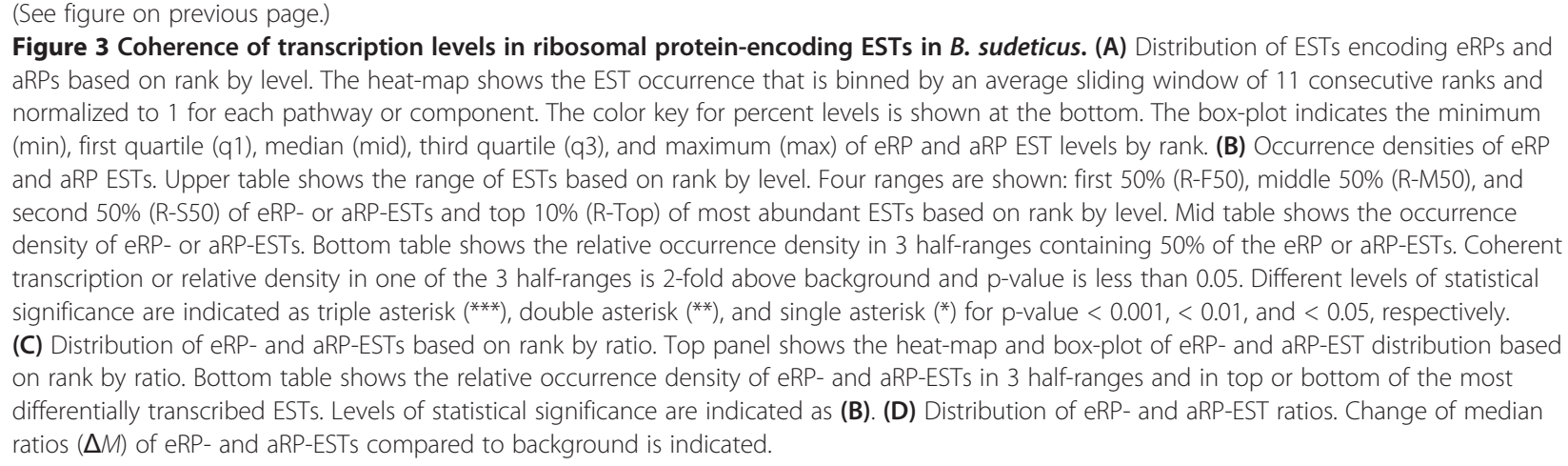

downregulated ESTs, indicating that photosynthesis is the most drastically downregulated pathway in B. sudeticus following ND.

Consistent with the downregulation of photosynthesisassociated ESTs, chlorophyll contents (i.e., chl a and b) in ND cells were also greatly reduced by 3.1 -fold compared to that of nitrogen-replete cells (i.e., $0.26 \pm 0.03 \%$ versus $0.81 \pm 0.11 \%$ of CDW, p-value $=0.001$ ) (Figure $5 \mathrm{D}$, upper panel). We subsequently determined the relative quantum yield or efficiency of photosystem II (i.e., Y(II) $=\left(F^{\prime}\right.$ '-F)/ Fm', see Methods), a measurement that is directly related to the quantum efficiency of carbon assimilation or photosynthesis [31]. Interestingly, photosynthesis efficiency was only reduced by $20 \%$ upon ND compared to nitrogen-replete conditions (i.e., $0.43 \pm 0.01$ versus 0 . $54 \pm 0.01$ ) (Figure 5D, bottom panel), not markedly reduced as seen in levels of chlorophyll content. Marginal reduction of quantum yield is consistent with the observation that cell mass increase and carbon assimilation were only slightly affected by ND stress (see Figure 1E). These results implied that photosynthetic components such as light harvesting proteins are in excess under optimal growth conditions. These proteins would be recycled for synthesis of new proteins required for gametogenesis and zygospore formation under ND conditions.

We investigated pathway-associated individual ESTs whose levels were greatly upregulated upon ND. From the 1,185 ESTs that were associated with 68 pathways, we found that 30 involved in 34 pathways were upregulated by 3-fold or greater (Additional file 6: Figure S1). Two lipid metabolism-specific enzymes, monoacylglycerol lipase (EC 3.1.1.23) and 3-oxoacyl-[acyl-carrier-protein] reductase (EC 1.1.1.100) were found. They might play a role in TAG accumulation upon ND.

\section{Abundant and coherent transcription of energy metabolic genes is conserved in actively growing $B$. sudeticus and $C$. reinhardtii cells}

We wanted to investigate if the basal transcription of energy metabolic genes was abundant and coherent in the model green microalga $C$. reinhardtii. For this reason, we obtained the transcription profiles of $C$. reinhardtii generated using Illumina technologies in the studies by Miller et al. and Gonzales-Ballester et al. [7,12]. Based on the best hits in C. reinhardtii, there were 4,148 $\mathrm{B}$. sudeticus ESTs whose best hits (or homologs) were available in transcriptomic datasets of $C$. reinhardtii $[7,12]$ (Additional file 7: Table S5). By using the common set of 4,148 ESTs, we found that correlation between basal transcription profiles in $B$. sudeticus and $C$. reinhardtii was moderate (i.e., correlation coefficient $=0.47 \sim 0.49$ ). The correlation between the two independent experiments in C. reinhardtii was relatively high (i.e., correlation coefficient $=0.76$ ), indicating a good quality of the two datasets (Figure 6A).

Based on the set of 4,148 ESTs, 53 metabolic pathways had 10 or more associated ESTs (i.e., due to the reduced number of ESTs, 13 pathways were filtered off from the list of 66 pathways based on the set of 7,625 ESTs; see Figures 4 and 5). We found 14 metabolic pathways and ribosomes (i.e., eRP and aRP) showed coherent transcription in rapidly growing log-phase B. sudeticus cells (Figure 6B; Additional file 8: Table S6; Additional file 9: Table S7). Though 5 of the 14 pathways were not previously shown when a set of 7,625 ESTs were used (see Figure 4B), the coherent pathways with high level of statistical significance (i.e., p-value < 0.001) remained unchanged.

To ensure a fair comparison, we applied a common set of 4,148 ESTs in B. sudeticus and C. reinhardtii. The relative occurrence density of the 53 metabolic pathways in 3 ranges (i.e., the first, middle, and second halves of the pathway-associated ESTs) was determined based on rank by level using the common set of 4,148 ESTs in B. sudeticus and $C$. reinhardtii (Figure 6B; Additional file 6: Figure S1).

Comparative analysis showed that eRP- and aRPencoding ESTs were both abundant and coherent in $B$. sudeticus and $C$. reinhardtii (Figure $6 \mathrm{C}$ ). Furthermore, we found that there were 10 metabolic pathways that were coherent in B. sudeticus and C. reinhardtii. Of those, 3, 2, and 5 involved in carbohydrate metabolisms, amino acid 


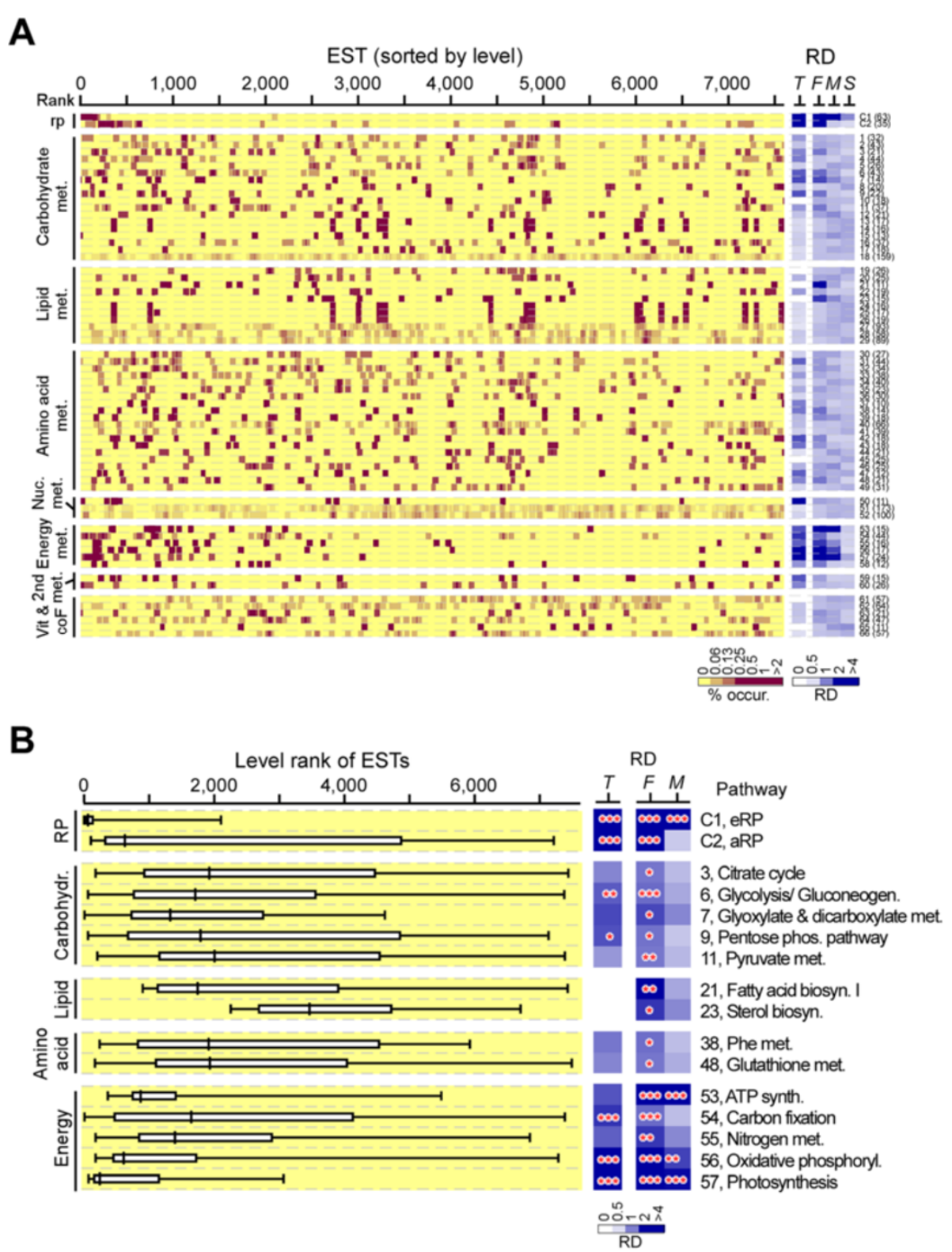

Figure 4 Coherence of transcription levels in various metabolic pathways in rapidly growing B. sudeticus cells. (A) Distribution of pathway-associated ESTs based on rank by level. Left panel shows the heat-map of individual EST occurrence based on rank by level. Classes of metabolic pathways are indicated on the far left. Numbers of ESTs associated with the pathway is shown in parentheses. RP stands for ribosomal proteins as control (e.g., C1 or C2). Right panel shows the relative occurrence density in the 3 half-ranges (F for first 50\%, M for middle 50\%, and S for second 50\%) of the pathway-associated ESTs and in the range of top $10 \%$ of the most abundant ESTs (i.e., T). Metabolic pathways are numbered on the far right. (B) Coherently transcribed metabolic pathways in rapidly growing B. sudeticus cells. Distribution of metabolic pathway-associated ESTs displaying the enriched level (i.e., 2-fold above background; $p$-value $<0.05$ ) in one of the 3 half-ranges $(F, M, o r S)$ is shown. Level of statistical significance is indicated by triple asterisk (**), double asterisk $\left(^{* *}\right)$, and single asterisk $\left(^{*}\right)$ for $p$-value $<0.001,0.01$, and 0.05 , respectively.

metabolisms, and energy metabolisms, respectively. We noted that 3 out of the 5 energy metabolic pathways were abundant in B. sudeticus. On the other hand, all 5 energy metabolic pathways were abundant (i.e., in one of the two experiments) in C. reinhardtii. In addition, 2 carbohydrate and 1 amino acid metabolic pathways were coherent and abundant in C. reinhardtii. Coherent pathways tended to be abundant in C. reinhardtii, but not in B. sudeticus.

We noted that 4 pathways were coherent in $B$. sudeticus but not in $C$. reinhardtii (Figure 6D). On the 
A

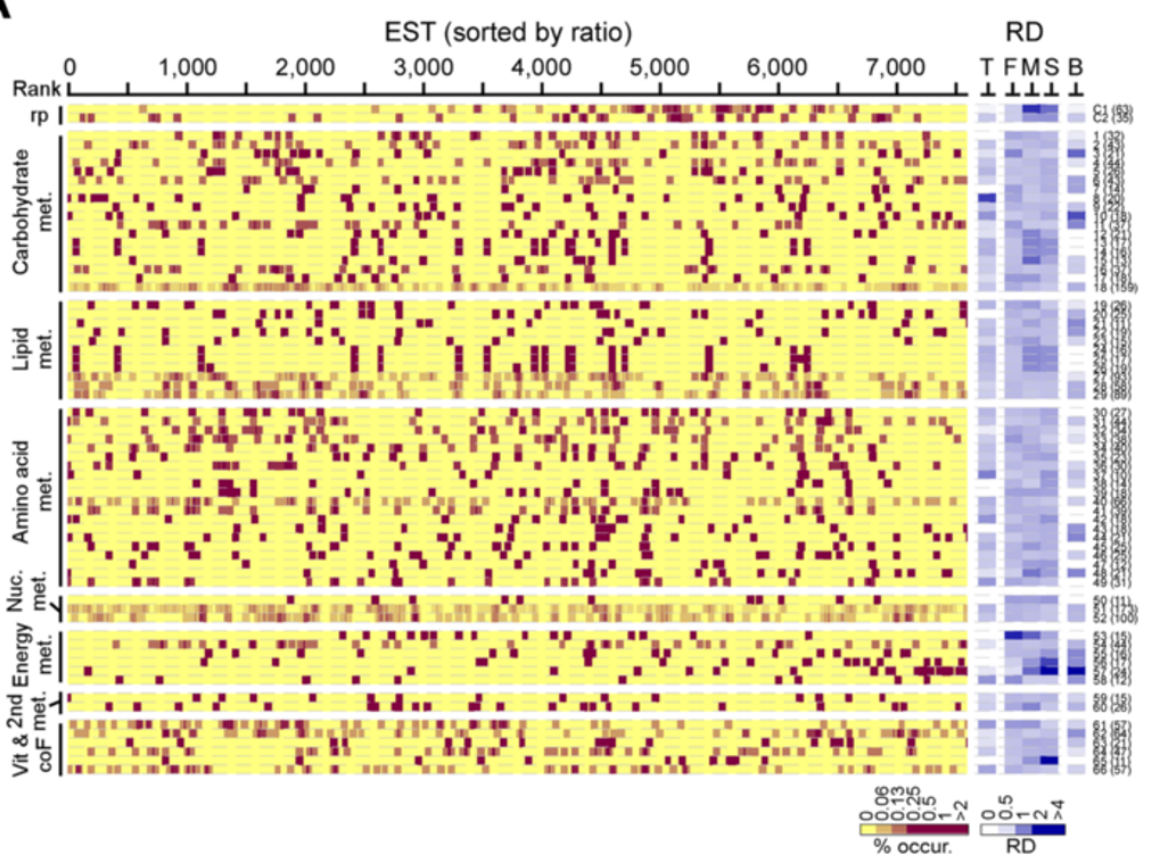

B

$\%$ occur $\mathrm{RD}$

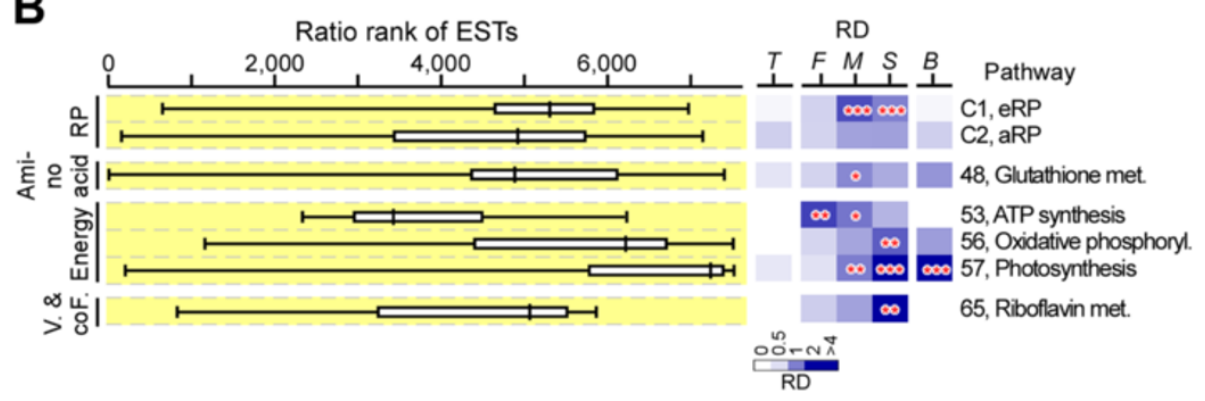

C

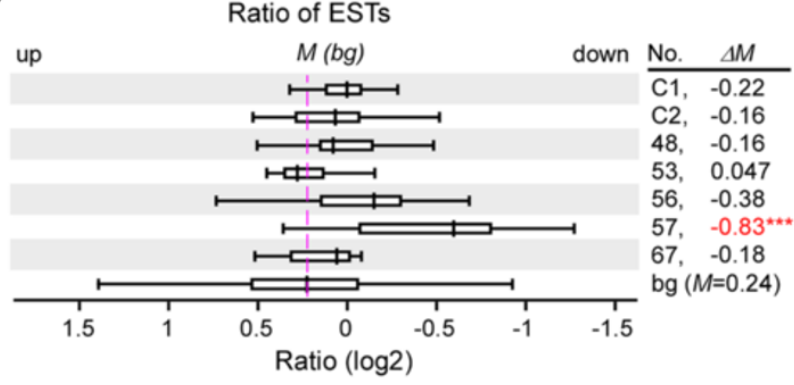

D

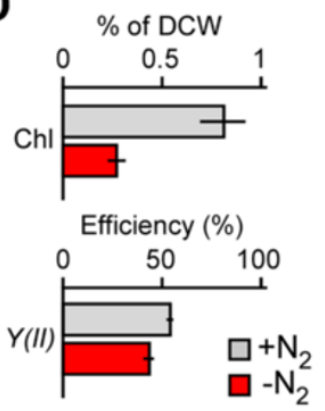

Figure 5 Coherence of transcriptional alterations in various metabolic pathways in B. sudeticus cells following ND. (A) Distribution of pathway-associated ESTs based on rank by ratio. The display is identical to Figure 4A. Relative occurrence density in the top10\% and bottom $10 \%$ of the most differentially transcribed ESTs is denoted as T and B, respectively. (B) Coherently responsive metabolic pathways in B. sudeticus cells following ND. The display is identical to Figure 4B. (C) Distribution of pathway-associated EST ratios. The display is identical to Figure 3D. Asterisk indicates the level of statistical significance. (D) Levels of chlorophyll content (upper panel) and efficiency of quantum yield of photosystem II (bottom panel). 


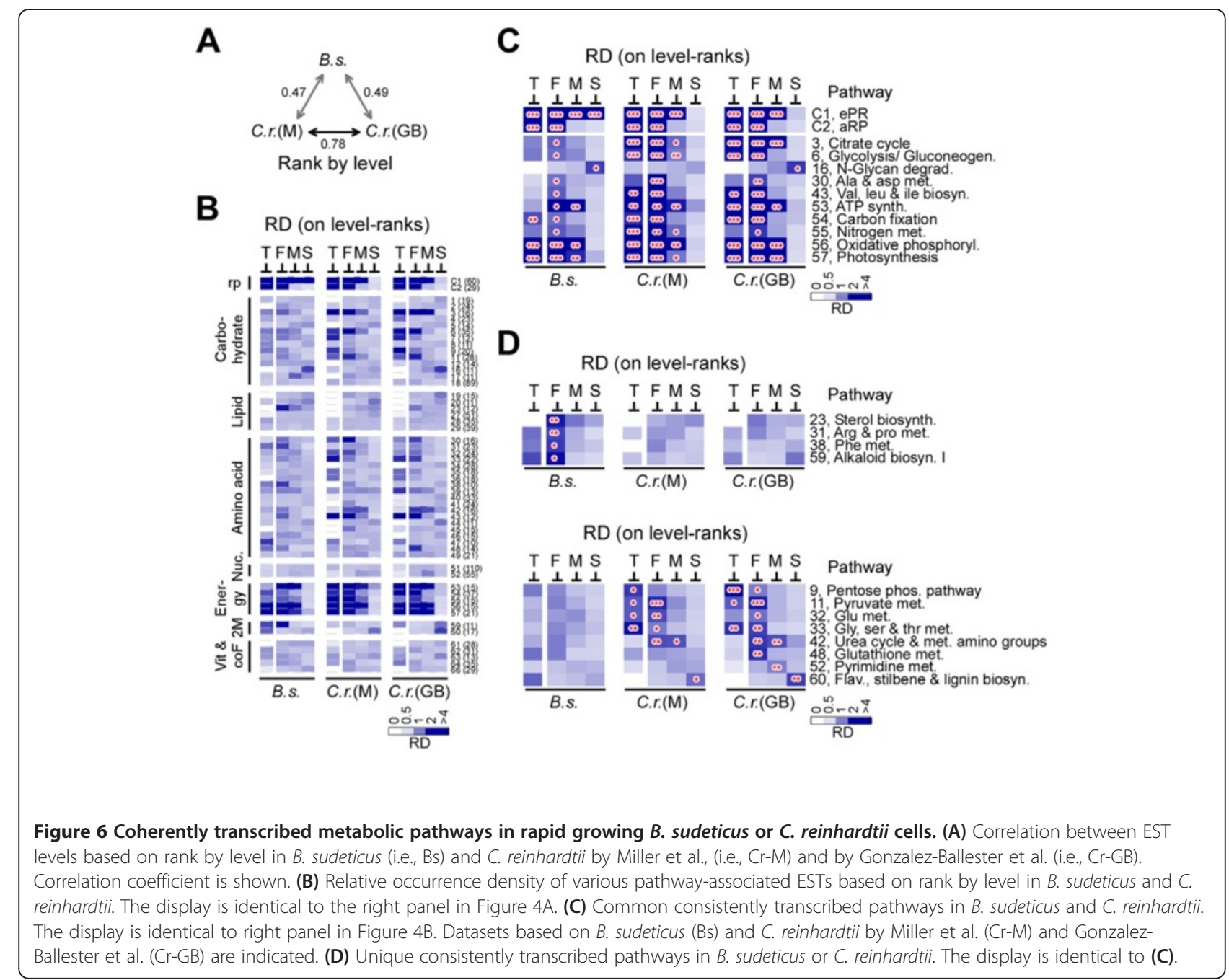

other hand, 8 were coherent in C. reinhardtii but not in B. sudeticus, 4 of which were also abundant. Interestingly, all coherent pathways were found to be involved in energy, carbohydrate, and amino acid metabolisms, but not in lipid metabolisms except for sterol biosynthesis in B. sudeticus.

\section{Transcriptional alteration of metabolic pathways but not individual ESTs is correlated in B. sudeticus and $C$. reinhardtii following ND}

Unlike the absolute level of ESTs, the relative level (or ratio between levels in ND and nitrogen-replete cells) of ESTs in B. sudeticus showed no correlation (i.e., correlation coefficient $=0.13$ ) with that of $C$. reinhardtii following ND based on the common set of 4,148 ESTs (Figure 7A). On the other hand, a moderate correlation (i.e., correlation coefficient $=0.42$ ) was observed between EST ratios in C. reinhardtii following ND and sulfur deprivation, suggesting that the global transcriptional response to ND was, to some extent, species-specific in green microalgae.

To compare the ND-induced transcription of ESTs associated with various metabolic pathways in B. sudeticus and C. reinhardtii, we determined the relative occurrence density in the 3 half-ranges of the pathway-associated ESTs based on rank by ratio (Figure 7B). We noted that median ratio of the pathway-associated ESTs in B. sudeticus was clearly correlated with that of $C$. reinhardtii following ND but not sulfur deprivation (Figure $7 \mathrm{C}$ ). This result suggested that transcriptional response of metabolic pathways rather than individual ESTs was conserved in green microalgae B. sudeticus and C. reinhardtii following ND.

In B. sudeticus, only eRP-ESTs but not aRP-ESTs showed coherent response to ND (Figure 7D). On the other hand, both eRP- and aRP-ESTs exhibited coherent response in $C$. reinhardtii. We noted that none of them displayed a significant alteration in transcriptional levels (based on the media) following ND in B. sudeticus and C. reinhardtii. Interestingly, though, the median level of eRP- or aRP- 


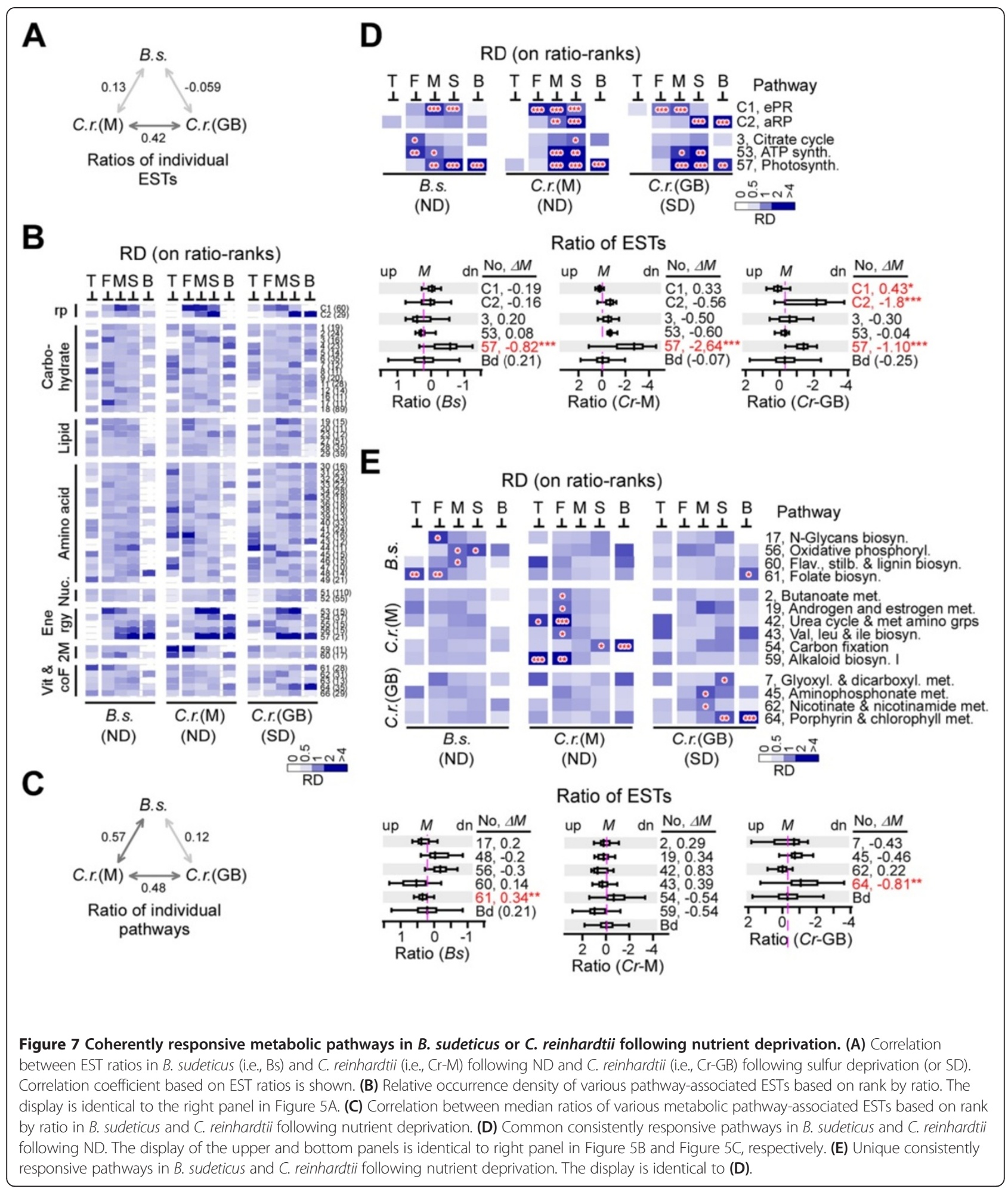

ESTs was significantly altered in C. reinhardtii following sulfur deprivation.

We found that there were 7 metabolic pathways exhibited consistent response (i.e., occurrence density in 1 of 3 half-ranges $>2$-fold; p-value $<0.05$ ) in B. sudeticus following ND, of which, 3 (i.e., citrate cycle, ATP synthesis, and photosynthesis) were also found to showed coherent response in $C$. reinhardtii in response to ND. While the median level of citrate cycle and ATP biosynthesis was not significantly changed, the median level of photosynthesis 
was obviously downregulated in B. sudeticus and $C$. reinhardtii following ND. In fact, it was also greatly downregulated in $C$. reinhardtii following sulfur deprivation. Consistent with it, aRP- but not eRP-ESTs were also downregulated in $C$. reinhardtii following sulfur deprivation. These results suggested that transcriptional downregulation of photosynthesis is a common response to nutrient deprivation.

Among the coherent pathways specific to species or stresses (Figure 7E), folate biosynthesis was upregulated (p-value < 0.01) in B. sudeticus following ND and porphyrin and chlorophyll metabolism was downregulated (p-value $<0.01$ ) in $C$. reinhardtii following sulfur deprivation. Folate and its derivatives are cofactors of onecarbon metabolism that is required for the biosynthesis of nucleotides and amino acids and upregulation of folate biosynthesis was in agreement with biosynthesis of novel proteins for gametogenesis upon ND. Chlorophyll is critical to photosynthesis. Hence, downregulation of porphyrin and chlorophyll metabolism was consistent with the repression of photosynthetic transcription.

\section{Discussion}

Based on the pathway-based approach, we show that the absolute level of ESTs encoding RPs and energy metabolic pathways such as photosynthesis, carbon fixation, and oxidative phosphorylation are highly coherent and abundant in B. sudeticus (see Figure 4 and Figure 6) and $C$. reinhardtii (see Figure 6) by using the analysis of EST occurrence density based on rank by level, implying that protein synthesis and energy metabolisms are the most active functions in the rapidly growing B. sudeticus and $C$. reinhardtii cells, possibly in all actively growing green microalgal cells. Interestingly, many coherent carbohydrate and amino acid metabolic pathways in C. reinhardtii but not in B. sudeticus tended to be abundant, probably reflecting the difference between $B$. sudeticus phototrophic growth and $C$. reinhardtii heterotrophic growth. We noted that almost all of the pathways whose coherence in EST steady-state levels occurred in the first 50\% (or the upper half) of the pathway-associated ESTs. It implies that there is a tight regulation of the abundantly expressed transcripts in microalgal cells. It would save energy for optimal function of complex metabolic pathways in microalgae.

ND is one of the most widely used methods for enhancement of storage lipids in green microalgae [4-10]. However, ND induces cell cycle arrest and sexual differentiation and spore formation for survival [11]. In this study, we show that photosynthesis is only metabolic pathway whose transcription is most downregulated in B. sudeticus following ND and C. reinhardtii following ND or sulfur deprivation (see Figure 7). In C. reinhardtii following sulfur deprivation, porphyrin and chlorophyll metabolism and aRP-ESTs are also downregulated. In plant, it is known that photosynthetic genes are downregulated in infected or damaged leaf by biotic agents such as arthropods, fungi, bacteria and viral pathogens [32]. Additionally, accumulation of carbohydrate under sink limitation can lead downregulation of photosynthesis in plant [33]. In unicellular green microalgae, it is not known if downregulation of photosynthesis is partly a result of carbohydrate (e.g., starch) accumulation in chloroplast.

In this study, we show that, upon ND, the level of nitrogen in cells is reduced by 2.5 -fold. While transcription of photosynthetic genes is drastically reduced, chlorophyll level is also reduced by 3 -fold. On the other hand, under ND conditions, cell mass still increases. Carbon content in ND cells is only decreased by $7 \%$ compared to that of nitrogen-replete cells. This is consistent with the observation that photosynthesis efficiency is only reduced by $20 \%$. These data indicate that drastic downregulation of photosynthetic transcripts or chlorophylls is primarily for recycling of nitrogen-rich proteins. It implies that photosynthetic proteins such as light-harvesting proteins are in excess in B. sudeticus, similar to large-sized antennabearing $C$. reinhardtii. It is found that cells with smallsized antenna in C. reinhardtii are more productive compared to that with large-sized antenna [34].

In yeast, genes encoding RPs and protein synthesis are downregulated in response to a number of stress factors including ND [35,36]. However, ribosome activity is not abolished. It is required for novel protein synthesis for preparation of sexual differentiation and zygospore formation. In mammalian systems, it has been shown that downregulation of protein synthesis is not mediated through dramatic repression of RP gene transcription but through reduced efficiency of translation [37]. It is possible that translation efficiency is decreased upon ND in green microalgae. This would explain the upregulation of eRP-ESTs in C. reinhardtii following sulfur deprivation (see Figure 7D). Downregulation of photosynthesis in algae following ND may provide a pool of nitrogen-rich molecules for synthesis of novel proteins required for adaptation and survival [38]. Given that most green microalgae resemble shade leaves having large-sized antenna, recycling of excessive photosynthetic proteins may be not immediately affect the photosynthesis capacity and carbon assimilation upon ND.

Transcriptional response to ND has been thoroughly studied in diatoms [39-41]. In Phaeodactylum tricornutum, carbon assimilation is found to continue after ND [39]. In addition, TAG content but not total lipid content is greatly increased following ND [39]. Noticeable reduction of photosynthesis activity (e.g., $20 \%$ of total activity) upon ND has also observed in diatom [42]. These results are very similar to what we have observed in B. sudeticus (in this study). 
ND induces lipid accumulation in B. sudeticus (Figures $1 C$ and 1D) and in C. reinhardtii $[4,6,7,9]$. However, no apparent up-regulation of lipid metabolic pathways based on KEGG database [43] is observed following ND in $B$. sudeticus and $C$. reinhardtii. This is consistent with the notion that regulation of storage lipid accumulation is complex in cells following ND: remodeling of membrane lipids for gametogenesis and accumulation of storage lipids through de novo biosynthesis and membrane lipid recycling [7]. Alternatively, regulation of TAG biosynthesis may involve post-translational modification [44]. Comparative analysis of global transcriptional response to ND in other microalgal species should provide insight into underlying molecular mechanisms of storage lipid accumulation following ND. Dissecting pathways responsible for lipid accumulation from those regulating sexual differentiation would allow construction of microalgal strains suitable for biomass and lipid production.

\section{Conclusions}

In this study, we utilized the commercial software CLC Genomics Workbench [16] to assemble the transcriptome without reference genome (see Methods). We show a clear correlation between transcription abundances of homologs in B. sudeticus and C. reinhardtii, suggesting a decent assembly of $B$. sudeticus transcriptome without reference genome using commercial software (see Figures 6 and 7). It provides a way for biologists to perform transcriptome studies in nonmodel organisms (i.e., no reference genome sequences) without much bioinformatics skills. By using the pathway-based approach, without performing time-course experiments, we are able to identify a number of probable gene regulatory networks (i.e., genes with coherent transcription in a pathway) that are involved in regulation of various metabolic pathways in rapidly growing or ND-induced B. sudeticus cells. Our result is useful for construction of motif-specific transcription regulatory networks when genome sequence is available. For transcriptome analysis involving a large numbers of samples such as in a timecourse experiment with multiple repeat, it is economical to use DNA microarray-based technologies. Hence, de novo assembled transcriptome is also useful in designing DNA microarray.

\section{Methods}

\section{Algal strains and culture manipulation}

The Botryosphaerella (or Botryococcus) sudeticus (UTEX2629) strain was obtained from the Culture Collection of Autotrophic Organisms (http://ccala.butbn.cas.cz/) with a strain catalog number of CCALA780 and the $C$. reinhardtii (UTEX89 or CC1009) strain was obtained from Chlamy Center (www.chlamy.org). CC1009 is a cell wall proficient and $\mathrm{NIT1}^{+}$and $\mathrm{NIT2}^{+}$strain. The cells were cultivated in $1 \mathrm{~L}$ of HS medium [25] or Bold's modified Bristol (BB) [26] medium in a $2.5 \mathrm{~L}$ low-form flask with shaking at $100 \mathrm{rpm}$ at $25^{\circ} \mathrm{C}$ under continuous illumination of $\sim 250 \mathrm{~mol}$ photon $\mathrm{m}^{-2} \mathrm{sec}^{-1}$. Culture was supplied with $2 \% \mathrm{CO}_{2}$ through bubbling. $\mathrm{BB}$ medium without sodium nitrate (or BB-N) was used as nitrogen-depleted medium.

Cell density of cultures was determined using cell dry weight $(\mathrm{CDW})$ in $\mathrm{g} / \mathrm{L}$. Approx. $50 \mathrm{ml}$ cell culture were harvested by filtration using the glass fiber filter GF/A (Whatman/GE Healthcare, Kent, UK) and dried in oven at $80^{\circ} \mathrm{C}$ overnight. CDW was measured in triplicate using the AG204 balance (Mettler-Toledo Inc., Columbus, OH).

The ratio of flagellates in cultures was determined by counting a total of 500 individual cells (e.g., flagellate or non-flagellate) in at least five independent fields in triplicate using Zeiss Axiovert $200 \mathrm{M}$ microscope (Carl Zeiss AG, Oberkochen, Germany).

To assess flocculation, shaken flasks (Corning Incorporated, Corning, NY, USA) were brought to standstill for $30 \mathrm{~min}$ to score for flocculation or sedimentation of cells at the bottom of flasks.

Log-phase culture in $\mathrm{BB}+\mathrm{N}$ medium at the $\mathrm{CDW}$ of $\sim 1.2$ $\mathrm{g} / \mathrm{L}$ is diluted by 8 -fold in fresh $\mathrm{BB}+\mathrm{N}$ and $\mathrm{BB}-\mathrm{N}$ medium and cultivated for 3 days as nitrogen-replete and nitrogendepleted cultures, respectively.

\section{Elemental analyses}

To determine nitrogen and carbon content of cells, approx. 3mg dry cell powder were subjected to elemental analysis using the the Vario Micro Cube (Elementar Analysensysteme GmbH, Hanau, Germany) in the Elemental Analysis Laboratory, Department of Chemistry, National University of Singapore. To determine nitrate in medium, $2 \mathrm{ml}$ culture supernatant was subjected to OD measurement using the Spectroquant Test Kit for Nitrate (Merck KGaA, Darmstadt, Germany) according to the manufacturer's instruction.

\section{Analyses of chlorophyll fluorescence and PAM- fluorescence of photosynthesis}

To determine chlorophyll content, cells from $2 \mathrm{ml}$ culture were harvested by centrifugation. The resulting cells were resuspended in 96\% ethanol and broken in the glass-bead beater FastPrep homogenizer (MP Biomedicals, Solon, $\mathrm{OH}$, USA). After incubation with ethanol for $2 \mathrm{~h}$ at $4^{\circ} \mathrm{C}$, cell debris was cleared by centrifugation. The resulting supernatant was subjected to OD measurement at the wavelength of $645 \mathrm{~nm}$ and $663 \mathrm{~nm}$, with the 96\% ethanol as blank. Total chlorophyll content (i.e., chl a and b) was estimated using a formula $\mathrm{C}(\mathrm{mg} / \mathrm{L})=20.2 \mathrm{OD}_{645}+8.05 \mathrm{D}_{663}$ [45] and was converted to \% of CDW.

To determine photosynthesis efficiency, cells were subjected to PAM-fluorescence analysis using the fluorometer 
Imaging-PAM (Heinz Walz GmbH, Effeltrich, Germany) according to the manufacturer's instruction. Briefly, the $\mathrm{Fm}^{\prime}$ and $\mathrm{F}$ were determined at the arctic light of $250 \mu \mathrm{mol}$ photon $\mathrm{m}^{-2} \mathrm{sec}^{-1}$ to mimic the growth conditions. The quantum yield was based on the formula $\mathrm{Y}(\mathrm{II})=$ (Fm'-F)//Fm' [46].

\section{Fluorescence microscopic analyses and fluorescence- activated cell sorting (FACS) analysis}

To examine the lipid content in B. sudeticus cells, $4 \mu \mathrm{l}$ of $0.25 \mathrm{mg} / \mathrm{ml}$ Nile red in acetone was directly added to 1 $\mathrm{ml}$ of fresh culture. After incubation at RT in dark for 15 min, fluorescence signals in B. sudeticus cells were examined using the Zeiss Axiovert $200 \mathrm{M}$ (Carl Zeiss AG, Oberkochen, Germany) with a Zeiss EC PlaneNeofluar 40x/0.75 objective lens. The images were captured by a CoolSNAP HQ monochrome digital camera (Roper Scientific, Ottobrunn, Germany) and processed using MetaMorph software (Molecular Devices, Sunnyvale, CA).

B. sudeticus cells were fixed with $10 \%$ formaldehyde $(\mathrm{w} / \mathrm{v})$ at RT for $15 \mathrm{~min}$. The cells were washed twice with $1 \times \mathrm{PBS}$ and stained by Nile red at the final concentration of $1 \mu \mathrm{g} / \mathrm{ml}$ for $15 \mathrm{~min}$ in dark. In microscopic analysis, Nile red stained cells were examined using the Zeiss Axiovert 200 M (Carl Zeiss AG, Oberkochen, Germany) with A Zeiss filter cube no. 15 (EX BP 546/12, BS FT 580, EM LP 590). In FACS analysis, Nile red stained cells were subjected to the analysis of BD FACS Calibur flow cytometer (Becton Dickinson Biosciences, San Jose, CA).

\section{TLC and GC-MS analysis}

Total lipid was exacted with methanol/ chloroform (2:1 by volume) solution and was quantified via gravimetric analysis after evaporation of solvents. Then it was dissolved in chloroform at a concentration of $0.1 \mathrm{mg} / \mu \mathrm{l}$. Equal amount of lipid was loaded on silica TLC plate (60F254, Merck Corporate, Whitehouse Station, NJ, USA) and developed in hexane/ diethyl ether/ acetic acid (35:15:0.1 by volume). TAG (tri-oleic acid (C18:1, [cis-9]) glyceride) and FFA (oleic acid (C18:1, [cis-9])) (Sigma-Aldrich, St. Louis, MO) was used as standard. Lipid profile on TLC plate was developed using iodine vapor. TAG and FAA contents were estimated through densitometric analysis of TLC profile. Hydrocarbons were exacted in equal amounts of total lipids with hexane. Hydrocarbons were analyzed using Shimadzu GC/MS-QP2010 Plus system (Shimadzu, Kyoto, Japan) equipped with a HP-5ms Ultra Inert column $(30 \mathrm{~m} \times 0.25 \mathrm{~mm} \times 0.25 \mu \mathrm{m}$, Agilent Technologies, Santa Clara, CA).

\section{Construction of CDNA libraries for next-generation sequencing analysis}

Total RNA was extracted from B. sudeticus cells using TRIzol $^{\circ}$ Plus RNA Purification System (Invitrogen-Life
Technologies Co., Carlsbad, CA, USA) according to manufacturer's protocol. Appr. $4 \mu \mathrm{g}$ of the resulting total RNA was used for synthesis of cDNA using the TruSeq RNA Sample Prep Kit (Invitrogen-Life Technologies Co.) according to manufacturer's instruction including synthesis of first and second strands cDNA, end repair, 3'-end adenylation, adapter ligation, fragment enrichment (e.g., $\sim 260$ bps in length), and library validation, quantification, and quality assessment with a bioanalyzer (Agilent Technologies; Santa Clara, CA, USA). The libraries are sequenced using the Illumina HiSeq 2000 Sequencer (BGI, Shenzhen, China).

\section{De novo assembly of Illumina short sequence reads}

Over 2 Giga-base clean paired-end reads $(90 \mathrm{bps}$ in length/ read) from each library were generated using HiSeq2000 technology. A total of 56 million reads from both growth conditions were pooled and subjected to de novo assembly using the CLC Genomics Workbench software (CLC Bio, Swansea, UK). As a result, 56 thousand nonredundant contigs/ scaffolds/ ESTs ranging from 200 to $15,500 \mathrm{bps}$ in length were obtained. More than $90 \%$ of total reads from each growth condition were mapped back to the 56 thousand ESTs, 99\% of which were found in both conditions. Read hits per EST were normalized to RPKM (Reads Per Kilobase per Million mapped reads) for estimation of ESTs transcription levels.

\section{Annotation of the $B$. sudeticus transcriptome}

All nonredundant ESTs were subjected to sequence homology comparison using the Basic Local Aliment Search Tool BLASTX suit against the "best" proteins in the model green microalgae $C$. reinhardtii and $C$. variabilis (genome. jgi-psf.org/Chlre4 and genome.jgi-psf.org/ChlNC64A_1). A total of 6,559 ESTs showed to have a best hit (i.e., expectation value $<1 \mathrm{E}-06)$ in the $C$. reinhardtii "best" proteins. Among the remaining ESTs, 1,066 showed to have a best hit (i.e., expectation value $<1 \mathrm{E}-06$ ) in the $C$. variabilis "best" proteins. To this end, 7,625 ESTs (13.6\%) in $B$. sudeticus were annotated based on the best hits in $C$. reinhardtii and C. variabilis (Additional file 2 Table S1). Of 7,625 ESTs, 4,860 were found to be associated with at least one of the Gene-ontology (GO) terms and 1,129 were associated with at least one of the Kyoto Encyclopedia of Genes and Genomes (KEGG) functions.

\section{Analysis of relative occurrence density of the pathway- associated ESTs}

Based on EST annotations, 66 (or 53) metabolic pathways were found to have 10 or more pathway-associated ESTs by using the set of 7,625 (or 4,148) ESTs (Additional file 5: Table S4). Average occurrence density (or background) of the pathway-associated ESTs is calculated as the ratio between numbers of pathway- 
associated ESTs and all ESTs tested. Occurrence density in the range containing first $50 \%$, middle $50 \%$, or second $50 \%$ of the pathway-associated ESTs is calculated as the ratio between $50 \%$ of the pathway-associated ESTs and all ESTs within the respective range. Relative occurrence density in a particular range is the ratio between the occurrence density in that range and the background.

Coherence of the constitutive transcription levels (or ND-induced transcription ratios) of various metabolic pathway-associated ESTs is defined as those whose relative occurrence density in one of the 3 half-ranges based on rank by level (or ratio) is 2-fold above background and $\mathrm{p}$-value $<0.05$. We assessed whether the coherent pathways were the most abundant (or most differently transcribed) ones in B. sudeticus by using the relative occurrence density in the top $10 \%$ of the most abundant (or most differentially transcribed) ESTs based on rank by level (or ratio) (i.e., >2-fold above background; pvalue $<0.05)$.

The distribution of pathway-associated ESTs based on ranks (i.e., level or ratio) in heat-map display is binned by an average sliding window of 11 consecutive ranks. The entire distribution of the ESTs in each pathway is normalized to 1 or $100 \%$.

\section{Comparative analysis between $B$. sudeticus and $C$. reinhardtii}

Of 7,625 annotated ESTs in B. sudeticus, 6,559 have a best hit in the $C$. reinhardtii "best" proteins. Based on the transcriptome analysis by Miller et al. [7] and Gonzalez-Ballester et al. [12], 4,148 out of 6,559 ESTs were available for comparisons. Therefore, coherence of transcriptions of 53 metabolic pathways was determined based on the set of 4,148 ESTs (Additional file 8: Table S6).

\section{Statistical analysis}

Binomial test was used to determine the statistical significance of enrichment for a number of pathway associated ESTs (i.e., observed) that are present within a range of ranked ESTs (i.e., trials). Average occurrence (or background level) of a pathway associated ESTs is the ratio between numbers of the pathway associated ESTs and all ESTs tested.

T-test was used to determine the statistical significance of level changes of pathway associated ESTs compared to all ESTs tested (or background). P-values are subjected to Bonferroni correction according to numbers of individual pathways tested against background.

The raw HiSEQ2000 pair-end sequencing data used in this study are available at the NCBI's Sequence Read Achieve (http://www.ncbi.nlm.nih.gov/sra) with the SRA Study accession number, SRA026047.

\section{Additional files}

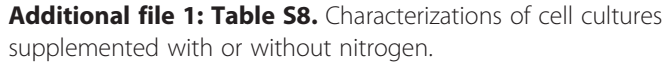

Additional file 2: Table S1. List of 7,625 annotated ESTs in B. sudeticus. Additional file 3: Table S2. List of EST-associated GO terms.

Additional file 4: Table S3. List of EST-associated KEGG metabolic pathways. Additional file 5: Table S4. List of 66 metabolic pathways and occurrence densities based on 7,625 ESTs.

Additional file 6: Figure S1. Upregulated (>3-fold) pathway-associated ESTs. Additional file 7: Table S5. List of 4,148 ESTs in B. sudeticus and C. reinhardtii.

Additional file 8: Table S6. List of 53 metabolic pathways and occurrence densities based on 4,148 ESTs.

Additional file 9: Table S7. Occurrence of genes based on ranks in various pathways.

\section{Competing interests}

The authors declare that they have no competing interests.

\section{Authors' contributions}

SD, LF, XZ carried out the algal biological studies. JZ and JL carried out the data analysis. YC performed part of the elemental analysis. JL conceived, designed, and supervised the study and drafted the manuscript. All authors read and approved the final manuscript.

\section{Acknowledgements}

The authors would like to thank P. Datta, J. Jeyakani, and N. Clark for assistances and advices on de novo transcriptome assembly. The authors are grateful to K. Nelson of JCVI and the anonymous reviewers for their comments that have greatly improved the manuscript. This work is partly supported by the $7^{\text {th }}$ JCO/A-STAR grant 11/03/FG/07/06 to $\mathrm{J}$ and $Y C$ and the $8^{\text {th }}$ JCO/A-STAR 1131CFG008 to JL. JZ, an undergraduate of Sichuan University, Chengdu, China, is an attachment student in GIS; LF and DS are supported by the post-doctoral fellowships under the JCO Grants 11/03/FG/ 07/06 and 1131CFG008, respectively.

\section{Author details}

${ }^{1}$ Systems Biology, Genome Institute of Singapore, 60 Biopolis Street, \#02-01, Singapore 138672, Singapore. ${ }^{2}$ Industrial Biotechnology, Institute of Chemical and Engineering Sciences, Singapore 627833, Singapore.

Received: 21 December 2012 Accepted: 7 October 2013

Published: 19 October 2013

\section{References}

1. Wijfels RH, Barbosa MJ: An outlook on microalgal biofuels. Science 2010, 329(5993):796-799.

2. Chisti Y: Biodiesel from microalgae beats bioethanol. Trends Biotechnol 2008, 26(3):126-131.

3. Microalgae biofuels and animal feeds. an introduction [http://www.scribd. com/doc/48492515/microalgae-biofuels-an-introduction-july23-2009benemann]

4. Hu Q, Sommerfeld M, Jarvis E, Ghirardi M, Posewitz M, Seibert M, Darzins A Microalgal triacylglycerols as feedstocks for biofuel production: perspectives and advances. Plant J 2008, 54(4):621-639.

5. Rodolfi L, Zittelli GC, Bassi N, Padovani G, Biondi N, Bonini G, Tredici MR: Microalgae for oil: strain selection, induction of lipid synthesis and out door mass cultivation in a low-cost protobioreactor. Biotechnol Bioeng 2009, 102:100-112.

6. Wang ZT, Ullrich N, Joo S, Waffenschmidt S, Goodenough U: Algal lipid bodies: stress induction, purification, and biochemical characterization in wild-type and starchless Chlamydomonas reinhardtii. Eukaryot Cell 2009, 8(12):1856-1868.

7. Miller R, Wu G, Deshpande RR, Vieler A, Gartner K, Li X, Moellering ER, Zauner S, Cornish AJ, Liu B, et al: Changes in transcript abundance in 
Chlamydomonas reinhardtii following nitrogen deprivation predict diversion of metabolism. Plant Physiol 2010, 154(4):1737-1752.

8. Stephenson AL, Dennis JS, Howe CJ, Scott SA, Smith AG: Influence of nitrogen-limitation regime on the production by Chlorella vulgaris of lipids for biodiesel feedstocks. Biogeosciences 2010, 1:47-58.

9. Work VH, Radakovits R, Jinkerson RE, Meuser JE, Elliott LG, Vinyard DJ, Laurens LM, Dismukes GC, Posewitz MC: Increased lipid accumulation in the Chlamydomonas reinhardtii sta7-10 starchless isoamylase mutant and increased carbohydrate synthesis in complemented strains. Eukaryot Cell 2010, 9(8):1251-1261.

10. Siaut M, Cuine S, Cagnon C, Fessler B, Nguyen M, Carrier P, Beyly A, Beisson F. Triantaphylides C, Li-Beisson $Y$, et al: Oil accumulation in the mode green alga Chlamydomonas reinhardtii: characterization, variability between common laboratory strains and relationship with starch reserves. BMC Biotechnol 2011, 11:7

11. Martin NC, Goodenough UW: Gametic differentiation in Chlamydomonas reinhardtii I. Production of gametes and their fine structure. J Cell Biol 1975, 67(3):587-605.

12. Gonzalez-Ballester D, Casero D, Cokus S, Pellegrini M, Merchant SS, Grossman AR: RNA-seq analysis of sulfur-deprived Chlamydomonas cells reveals aspects of acclimation critical for cell survival. Plant Cell 2010, 22(6):2058-2084

13. Merchant SS, Prochnik SE, Vallon O, Harris EH, Karpowicz SJ, Witman GB, Terry A, Salamov A, Fritz-Laylin LK, Marechal-Drouard L, et al: The Chlamydomonas genome reveals the evolution of key animal and plant functions. Science 2007, 318(5848):245-250.

14. Blanc G, Duncan G, Agarkova I, Borodovsky M, Gurnon J, Kuo A, Lindquist E, Lucas S, Pangilinan J, Polle J, et al: The Chlorella variabilis NC64A genome reveals adaptation to photosymbiosis, coevolution with viruses, and cryptic sex. Plant Cell 2010, 22(9):2943-2955.

15. De Wit P, Pespeni MH, Ladner JT, Barshis DJ, Seneca F, Jaris H, Therkildsen NO, Morikawa M, Palumbi SR: The simple fool's guide to population genomics via RNA-Seq: an introduction to high-throughput sequencing data analysis. Mol Ecol Resour 2012, 12(6):1058-1067.

16. Brautigam A, Mullick T, Schliesky S, Weber AP: Critical assessment of assembly strategies for non-model species mRNA-Seq data and application of next-generation sequencing to the comparison of $C(3)$ and C(4) species. J Exp Bot 2011, 62(9):3093-3102.

17. Compeau PE, Pevzner PA, Tesler G: How to apply de Bruijn graphs to genome assembly. Nat Biotechnol 2011, 29(11):987-991.

18. Grabherr MG, Haas BJ, Yassour M, Levin JZ, Thompson DA, Amit I, Adiconis X, Fan L, Raychowdhury R, Zeng Q, et al: Full-length transcriptome assembly from RNA-Seq data without a reference genome. Nat Biotechnol 2011, 29(7):644-652.

19. Rismani-Yazdi H, Haznedaroglu BZ, Bibby K, Peccia J: Transcriptome sequencing and annotation of the microalgae Dunaliella tertiolecta: pathway description and gene discovery for production of nextgeneration biofuels. BMC Genomics 2011, 12:148.

20. Guarnieri MT, Nag A, Smolinski SL, Darzins A, Seibert M, Pienkos PT: Examination of triacylglycerol biosynthetic pathways via de novo transcriptomic and proteomic analyses in an unsequenced microalga. PLoS One 2011, 6(10):e25851.

21. Rismani-Yazdi H, Haznedaroglu BZ, Hsin C, Peccia J: Transcriptomic analysis of the oleaginous microalga Neochloris oleoabundans reveals metabolic insights into triacylglyceride accumulation. Biotechnol Biofuels 2012, 5(1):74.

22. Vazquez-Duhalt R, Greppin H: Growth and production of cell constituents in batch cultures of Botryococcus sudeticus. Phytochem 1987, 26:885-890.

23. Senousy HH, Beakes GW, Hack E: Phylogenetic placement of Botryococcus braunii (Trebouxiophyceae) and Botryococcus sudeticus isolate UTEX 2629 (Chlorophyceae). J Phycol 2004, 40:412-423.

24. Přibyl P, Cepák V: Evidence for sexual reproduction and zoospore formation in Botryosphaerella sudetica UTEX 2629, previously assigned to the genus Botryococcus (Chlorophyceae, Chlorophyta). Nova Hedwigia 2007, 85:63-71.

25. Sueoka N: Mitotic Replication of Deoxyribonucleic Acid in Chlamydomonas Reinhardi. Proc Natl Acad Sci U S A 1960, 46(1):83-91.

26. Bold HC: The morphology of Chlamydomonas chlamydogama sp. Bull Torrey Bot Club 1949, 76:101-108.

27. Ashburner M, Ball CA, Blake JA, Botstein D, Butler H, Cherry JM, Davis AP, Dolinski K, Dwight SS, Eppig JT, et al: Gene ontology: tool for the unification of biology The Gene Ontology Consortium. Nat Genet 2000, 25(1):25-29.

28. Kanehisa M, Goto S, Sato Y, Furumichi M, Tanabe M: KEGG for integration and interpretation of large-scale molecular data sets. Nucleic Acids Res 2012, 40:D109-114.

29. Warner JR: The economics of ribosome biosynthesis in yeast. Trends Biochem Sci 1999, 24(11):437-440.

30. Berg JM, Tymoczko JL, Stryer L: Biochemistry. 5th edition. New York: W H Freeman; 2002.

31. Baker NR: Chlorophyll fluorescence: a probe of photosynthesis in vivo. Annu Rev Plant Biol 2008, 59:89-113.

32. Bilgin DD, Zavala JA, Zhu J, Clough SJ, Ort DR, DeLucia EH: Biotic stress globally downregulates photosynthesis genes. Plant Cell Environ 2010, 33(10):1597-1613.

33. Nebauer SG, Renau-Morata B, Guardiola JL, Molina RV: Photosynthesis down-regulation precedes carbohydrate accumulation under sink limitation in Citrus. Tree Physiol 2011, 31(2):169-177.

34. Polle JE, Kanakagiri SD, Melis A: tla1, a DNA insertional transformant of the green alga Chlamydomonas reinhardtii with a truncated light-harvesting chlorophyll antenna size. Planta 2003, 217(1):49-59.

35. Gasch AP, Spellman PT, Kao CM, Carmel-Harel O, Eisen MB, Storz G, Botstein D, Brown PO: Genomic expression programs in the response of yeast cells to environmental changes. Mol Biol Cell 2000, 11(12):4241-4257.

36. Causton HC, Ren B, Koh SS, Harbison CT, Kanin E, Jennings EG, Lee TI, True HL, Lander ES, Young RA: Remodeling of yeast genome expression in response to environmental changes. Mol Biol Cell 2001, 12(2):323-337.

37. Ceppi M, Clavarino G, Gatti E, Schmidt EK, de Gassart A, Blankenship D, Ogola G, Banchereau J, Chaussabel D, Pierre P: Ribosomal protein mRNAs are translationally-regulated during human dendritic cells activation by LPS. Immunome Res 2009, 5:5.

38. Collier JL, Grossman AR: Chlorosis induced by nutrient deprivation in Synechococcus sp. strain PCC 7942: not all bleaching is the same. J Bacteriol 1992, 174(14):4718-4726.

39. Valenzuela J, Mazurie A, Carlson RP, Gerlach R, Cooksey KE, Peyton BM, Fields MW: Potential role of multiple carbon fixation pathways during lipid accumulation in Phaeodactylum tricornutum. Biotechnol Biofuels 2012, 5(1):40.

40. Mock T, Samanta MP, Iverson V, Berthiaume C, Robison M, Holtermann K, Durkin C, Bondurant SS, Richmond K, Rodesch M, et al: Whole-genome expression profiling of the marine diatom Thalassiosira pseudonana identifies genes involved in silicon bioprocesses. Proc Natl Acad Sci U S A 2008, 105(5):1579-1584.

41. Hockin NL, Mock T, Mulholland F, Kopriva S, Malin G: The response of diatom central carbon metabolism to nitrogen starvation is different from that of green algae and higher plants. Plant Physiol 2012, 158 (1):299-312.

42. Yang ZK, Niu YF, Ma YH, Xue J, Zhang MH, Yang WD, Liu JS, Lu SH, Guan Y, Li HY: Molecular and cellular mechanisms of neutral lipid accumulation in diatom following nitrogen deprivation. Biotechnol Biofuels 2013, 6(1):67.

43. Kanehisa M, Goto S, Kawashima S, Okuno Y, Hattori M: The KEGG resource for deciphering the genome. Nucleic Acids Res 2004, 32:D277-280.

44. Holm C, Osterlund T, Laurell H, Contreras JA: Molecular mechanisms regulating hormone-sensitive lipase and lipolysis. Annu Rev Nutr 2000, 20:365-393

45. Sartory DR, Grobbelaar JU: Extraction of Chlorophyll a from Freshwater Phytoplankton for Spectrophotometric Analysis. Hydrobiologia 1984, 114:177-187.

46. Klughammer C, Schreibe U: Complementary PS II quantum yields calculated from simple fluorescence parameters measured by PAM fluorometry and the Saturation Pulse method. PAM Application Notes 2008, 1:27-35.

doi:10.1186/1471-2164-14-715

Cite this article as: Sun et al:: De novo transcriptome profiling uncovers a drastic downregulation of photosynthesis upon nitrogen deprivation in the nonmodel green alga Botryosphaerella sudeticus. BMC Genomics $201314: 715$ 